\title{
Modeling Network Traffic by a Cluster Poisson Input Process with Heavy and Light-Tailed File Sizes
}

\author{
Vicky Fasen*
}

July 4, 2010

\begin{abstract}
We consider a cluster Poisson model with heavy-tailed interarrival times and cluster sizes as a generalization of an infinite source Poisson model where the file sizes have a regularly varying tail distribution function or a finite second moment. One result is that this model reflects long range dependence of teletraffic data. We show that depending on the heaviness of the file sizes, the interarrival times and the cluster sizes we have to distinguish different growths rates for the time scale of the cumulative traffic. The mean corrected cumulative input process converges to a fractional Brownian motion in the fast growth case. However, in the intermediate and the slow growth case we can have convergence to a stable Lévy motion or a fractional Brownian motion as well depending on the heaviness of the underlying distributions. These results are contrary to the idea that cumulative broadband network traffic converges in the slow growth case to a stable process. Furthermore, we derive the asymptotic behavior of the cluster Poisson point process which models the arrival times of data packets and the individual input process itself.
\end{abstract}

AMS 2000 Subject Classifications: primary: 90B22 secondary: 60F05, 60F10, 60F17, 60G52

Keywords: cluster Poisson model, covariance function, cumulative input process, fluid queue, fractional Brownian motion, heavy tails, input model, long range dependence, self-similarity, stable Lévy motion, regular variation, scaling, teletraffic.

${ }^{*}$ Center for Mathematical Sciences, Technische Universität München, D-85747 Garching, Germany, email: fasen@ma.tum.de. 


\section{Introduction}

The infinite source Poisson model is a fluid queue approximation of network data traffic that assumes that the transmission of data files to the system happens at the jump times of a Poisson process. The file sizes are assumed to form an iid (independently and identically distributed) sequence independent of the underlying Poisson process. This model is also called $M / G / \infty$ model in the queueing literature. There is empirical evidence that the file sizes are heavy-tailed with an index of regular variation in the interval $(1,2)$ (cf. $[7,8,9,18,35]$ ). The infinite variance of the file size in the infinite source Poisson model causes long range dependence and self-similarity in the input process which are stylized facts of teletraffic data (cf. the data analysis in $[7,8,9,22]$ ). However, this explains the long-range dependence of one individual source, but not that of the overall teletraffic. The teletraffic of the network was studied in the seminal work of Mikosch et al. [25]; see also the monographs of Resnick [30] and Whitt [34]. Guerin et al. [17] applied the infinite source Poisson model to teletraffic data and realized some shortcomings in that model. They suggested as an alternative a cluster Poisson model to be more realistic.

A cluster Poisson model is an obvious extension of the infinite source Poisson model. In the present paper we consider a cluster Poisson point process $N$ modeling the arrival of data files sent to a server, i.e. we have a Poisson point process where at any jump time a cluster of data transmissions is initiated. The cluster sizes will have a finite first moment but an infinite second moment, and the interarrival times have an infinite second moment as well. This causes $N$ to be long range dependent and makes $N$ sufficiently irregular for our results (see Section 3).

On the other hand, we consider the amount of data which is transmitted to the server, the so called input process $(A(t))_{t \geq 0}$. The file sizes are assumed to be regularly varying of index $\gamma \in(1,2)$ or have a finite second moment. Hence, the present paper contains also the model of Fasen and Samorodnitsky [15] and extends their results. In particular, we are interested in the characterization of the traffic generated by one source, but on the other hand we are also concerned with the overall traffic of the network and on which way the properties of an individual source are transferred to the traffic of the system. The teletraffic of the network is given by the cumulative input of all sources. Thus, let $\left(A_{i}\right)_{i \in \mathbb{N}}$ be iid input processes; e.g. independent users in a computer pool generate independent traffic and $A_{i}$ models the traffic of user $i$. With $n$ input processes at a time scale $M$, the mean corrected cumulative input is the stochastic process

$$
S_{n, M}(t)=\sum_{i=1}^{n}\left(A_{i}(M t)-\mathbb{E}\left(A_{i}(M t)\right)\right) \quad \text { for } t \geq 0 .
$$

The case where the time scale $M=M_{n}$ depends on the number of users $n$ (bird's eye view) is the situation where the term fast growth and slow growth case comes in. Let $\left(M_{n}\right)$ be a sequence of positive constants such that $M_{n} \uparrow \infty$ as $n \uparrow \infty$. Then the fast growth case means $n \gg M_{n}$ while the slow growth case means $n \ll M_{n}$. In this paper we will further use an intermediate growth case; see Section 5.2. Then $\left(M_{n}^{(1)}\right)$ and $\left(M_{n}^{(2)}\right)$ are both sequences of positive constants tending to $\infty$ as $n \rightarrow \infty$ with $M_{n}^{(1)}<<M_{n}^{(2)}$ where $n<<M_{n}^{(1)}$ reflects the slow, $M_{n}^{(1)}<<n<<M_{n}^{(2)}$ the intermediate and $n>>M_{n}^{(2)}$ the fast growth case. In Gaigalas and Kaj [16], who studied 
the renewal counting process of Pipiras and Taqqu [28] with heavy-tailed inter-renewal times as a packet arrival model, an intermediate growth case also exist. However, their definition of intermediate growth case is different to ours.

For a cluster Poisson model we can interpret the different growth cases also as a change in the connection rate of one source, since the input of one cluster Poisson model with intensity $\lambda(n)=n \lambda_{0}$ has the same distribution as the cumulative input of $n$ iid cluster Poisson sources with intensity $\lambda_{0}$. Thus, Mikosch et al. [25] described their growth rates of $\left(M_{n}\right)$ by a change in the connection rate rather than in the change of the number of users.

There are substantial reasons to separate the growth cases. If the number of sources $n$ is very large, then the process in (1.1) is the sum of a very large number of iid terms that change relatively slowly. Hence, under general assumptions we can apply a central limit theorem and obtain convergence to a Gaussian process; see the interesting paper of Mikosch and Samorodnitsky [26]. On the other hand, if the time scale $M$ is very large, then the main phenomenon in (1.1) is, actually, in the deviations of the individual input processes from their means, $A(M t)-\mathbb{E}(A(M t))$, for large $M$. Then it is not obvious which limit we will obtain. In Levy and Taqqu $[32,23,24]$ and Pipiras and Taqqu [28] we find these different cases of first letting $n$ and then $M$ tend to infinity and opposite for a renewal-reward model. Their limit process not only depends on the order of the limits but also on whether the tails of the rewards are lighter or heavier than those of the renewals; fractional Brownian motion, stable Lévy motion as well as stable processes with dependent increments are possible limits (cf. Kaj and Taqqu [19] for an extension of that idea). We will see a similar phenomena in our model.

We show that the asymptotic behavior of the variance of the input process and the asymptotic behavior of the mean corrected individual input process

$$
A_{n}(t)=\frac{A\left(0, M_{n} t\right]-\mathbb{E}\left(A\left(0, M_{n} t\right]\right)}{a_{n}}, \quad t \geq 0,
$$

respectively, where $\left(a_{n}\right)$ is a sequence of positive constants tending to $\infty$, is either determined by the cluster Poisson point process $N$, if $\gamma>3-2 H\left(\gamma>H^{-1}\right.$, respectively) and if the file sizes have a finite second moment, or by the file sizes if $\gamma<3-2 H\left(\gamma<H^{-1}\right.$, respectively), where $H$ is a constant defined by the cluster Poisson model. We use these results on the traffic of one source to mathematically explain the behavior of the teletraffic in the system. Thus, we illustrate that the different regions for $\gamma$ in the asymptotic behavior of the variance and the input process have also to be distinguish in the asymptotic behavior of the mean corrected cumulative input of the network. If $\gamma>3-2 H$ or if the file sizes have finite second moment, the process $\left(S_{n, M_{n}}(t)\right)_{t \geq 0}$ converges for any (sufficiently regular) sequence $M_{n} \uparrow \infty$ as $n \rightarrow \infty$ to a fractional Brownian motion. This result extends the results of Fasen and Samorodnitsky [15] who assumed finite second moments of the file sizes. On the one hand, we also allow heavy-tailed file sizes and on the other hand, our results include the fast growth case. However, the result is surprising. In the slow growth case of an ON/OFF and an infinite source Poisson model with heavy-tailed file sizes the limit is a stable Lévy motion (cf. Mikosch et al. [25]). Mikosch and Samorodnitsky [26] discovered that the occurrence of a stable limit, in the slow growth case of a 
general telecommunication model, is not robust. Our result confirms that idea.

In contrast, for $H^{-1}<\gamma<3-2 H$ we have to distinguish three different growth rates of $\left(M_{n}\right)$ where any growth case has a different scaling and limit distribution; a fractional Brownian motion with index $(3-\gamma) / 2$ or $H$, or a $\gamma$-stable Lévy motion. Again we obtain in the slow growth case the convergence to a fractional Brownian motion and not to a stable process. Finally, the case $\gamma<H^{-1}$ recovers the classical result of the ON/OFF and the infinite source Poisson model; in the fast growth case the convergence to a fractional Brownian motion and in the slow growth case to a $\gamma$-stable Lévy motion.

The paper is organized as follows. We start with some preliminaries in Section 2. In Section 2.1 we introduce a general cluster Poisson model and in Section 2.2 we present the model assumptions. As mentioned, a typical stylized fact of teletraffic data is long range dependence. Hence, in Section 3 we compute the asymptotic behavior of the variance and the covariance of the input process $A$ and the point process $N$. On the one hand, we obtain that the variance behaves asymptotically like a power function of some index in $(0,1)$, and hence $N$ and $A$ exhibit long range dependence. On the other hand, we also see the influence of the underlying point process $N$ and the file sizes $Z$ on the dependence structure of $A$. Next, we study the asymptotic properties of

$$
\frac{N\left(0, M_{n} t\right]-\mathbb{E}\left(N\left(0, M_{n} t\right]\right)}{\sqrt{\operatorname{Var}\left(N\left(0, M_{n}\right]\right)}} \quad \text { for } t \geq 0,
$$

in Section 4. The results are different to the conclusions for the cluster Poisson point process in Faÿ et al. [14] depending on their different model assumptions. Independent of the growth rate of $\left(M_{n}\right)$ we obtain the convergence of (1.3) to a fractional Brownian motion. Finally, the asymptotic behavior of the input process $\left(A_{n}(t)\right)_{t \geq 0}$ and the properly normalized cumulative input $\left(S_{n, M_{n}}(t)\right)_{t \geq 0}$ are derived in Section 5 . To increase the readability of the paper the proofs are postponed to Section 6 .

We will use the notation $\Longrightarrow$ for weak convergence, $\stackrel{\mathbb{P}}{\longrightarrow}$ for convergence in probability, $\stackrel{\nu}{\Longrightarrow}$ for vague convergence, and $\stackrel{\text { FDD }}{\Longrightarrow}$ for weak convergence of the finite dimensional distributions. For $x \in \mathbb{R}$ we write $x_{+}=\max (0, x)$ and $\lceil x\rceil=\inf \{k \in \mathbb{N}: k \geq x\}$. For two random variables $X, Y$ the symbol $X \stackrel{d}{=} Y$ means that $X$ has the same distribution as $Y$. Let $\left(x_{n}\right)_{n \in \mathbb{N}},\left(y_{n}\right)_{n \in \mathbb{N}}$ be sequences of constants then $x_{n} \sim y_{n}$ as $n \rightarrow \infty$ iff $\lim _{n \rightarrow \infty} x_{n} / y_{n}=1$. For a distribution function $F$ we denote by $\bar{F}=1-F$ the tail of $F$ and finally, Leb denotes the Lebesgue measure.

\section{Preliminaries}

\subsection{The cluster Poisson model}

A cluster Poisson point process, which we use to model the arrival times of data files sent to the server, is defined as follows:

(i) The initial cluster points, denoted by $\ldots<\Gamma_{-1}<\Gamma_{0}<0<\Gamma_{1}<\Gamma_{2}<\ldots$ form a homogeneous Poisson process with rate $\lambda_{0}$, the connection rate. 
(ii) Each cluster initiated at $\Gamma_{m}$ is of random size $\left(K_{m}+1\right)$ with arrival points

$$
\Gamma_{m, k}=\Gamma_{m}+T_{m, k} \quad \text { for } k=0, \ldots, K_{m}
$$

where

$$
T_{m, k}=\sum_{j=1}^{k} X_{m, j} \text { for } k \in \mathbb{N} \quad \text { and } \quad T_{m, 0}=0,
$$

and the within-cluster arrival times $\left(X_{m, j}\right)_{j, m \in \mathbb{N}}$ are a sequence of iid positive random variables with common distribution $F_{X}$ independent of the sequence of iid positive cluster sizes $\left(K_{m}\right)_{m \in \mathbb{Z}}$ with distribution $F_{K}$.

Note that $K=K_{m}=0$ gives us the infinite source Poisson model. The times $\left(\Gamma_{m, k}\right)$ correspond in a telecommunication model with the arrival times of data files, denoted by $\left(Z_{m, k}\right)$, at the server. The cluster Poisson point process describing the arrivals of data files is then

$$
N=\sum_{m \in \mathbb{Z}} \sum_{k=0}^{K_{m}} \varepsilon_{\Gamma_{m, k}}
$$

where $\varepsilon$ denotes the dirac measure. This means $N(s, t]$ counts the number of arrivals of data packets in the time interval $(s, t]$. If $\mathbb{E}(K)<\infty$ then by Faÿ et al. [14], Proposition 2.1, the intensity of $N$ is given by

$$
\lambda=\lambda_{0}(1+\mathbb{E}(K))
$$

Mostly we will neglect the index $m$ and write shortly

$$
T_{k}=\sum_{j=1}^{k} X_{j}, \quad k \in \mathbb{N},
$$

for an iid sequence $\left(X_{j}\right)$ with distribution $F_{X}$. Further, we assume:

(iii) the file sizes $\left(Z_{m, k}\right)_{m, k \in \mathbb{N}_{0}}$ are again an iid sequence of positive random variables with distribution function $F_{Z}$ and $\mathbb{E}|Z|<\infty$;

(iv) $\left(\Gamma_{m, k}\right),\left(K_{m}\right),\left(Z_{m, k}\right)$ and $\left(X_{j, m}\right)$ are independent sequences.

Here,

$$
N^{(M)}=\sum_{m \in \mathbb{Z}} \sum_{k=0}^{K_{m}} \varepsilon_{\left(\Gamma_{m, k}, Z_{m, k}\right)}
$$

is a stationary marked point process. For details on stationary marked point processes we refer to Karr [21], Daley and Vere-Jones [10, 11] and Faÿ et al. [14]. 
If $\left(\mathcal{T}_{l}\right)_{l \in \mathbb{Z}}$ denotes the ordered arrival times of $\left(\Gamma_{m, k}\right)_{m \in \mathbb{Z}, k=0, \ldots, K_{m}}$ with corresponding file sizes $\left(\mathcal{Z}_{l}\right)_{l \in \mathbb{Z}}$ where $\left(\mathcal{Z}_{l}\right)_{l \in \mathbb{Z}}$ is again an iid sequence with distribution $F_{Z}$, then the point processes $N$ and $N^{(M)}$ have the representation

$$
N=\sum_{l \in \mathbb{Z}} \varepsilon_{\mathcal{T}_{l}} \quad \text { and } \quad N^{(M)}=\sum_{l \in \mathbb{Z}} \varepsilon_{\left(\mathcal{T}_{l}, \mathcal{Z}_{l}\right)}
$$

where $\ldots<\mathcal{T}_{-1}<\mathcal{T}_{0}<0<\mathcal{T}_{1}<\ldots$ as well.

Now the amount of data brought to the server is modeled by the input process

$$
A(t)=\sum_{m \in \mathbb{Z}} \sum_{k=0}^{K_{m}}\left[Z_{m, k} \wedge\left(t-\Gamma_{m, k}\right)_{+}-Z_{m, k} \wedge\left(-\Gamma_{m, k}\right)_{+}\right]=\int_{\mathbb{R} \times \mathbb{R}_{+}} f d N^{(M)},
$$

where

$$
f(s, z)=\int_{0}^{t} \mathbf{1}_{\{s<x<s+z\}} d x \quad \text { for }(s, z) \in \mathbb{R} \times \mathbb{R}_{+} .
$$

With that representation we are able to compute the first and second moments of $A(t)$ using the Poisson structure of the underlying point process $N^{(M)}$ and hence, of $A$. This is presented in the next lemma whose proof is postponed to Section 6 .

\section{Lemma 2.1}

(a) Let $f: \mathbb{R} \times \mathbb{R}_{+} \rightarrow \mathbb{R}$ be measurable. Then

$$
\mathbb{E}\left(\int_{\mathbb{R}^{\prime} \mathbb{R}_{+}} f d N^{(M)}\right)=\lambda \int_{\mathbb{R}^{\prime} \times \mathbb{R}_{+}} f(s, z) F_{Z}(d z) d s .
$$

(b) Let $f, g: \mathbb{R} \rightarrow \mathbb{R}_{+}$be measurable. Then

$$
\operatorname{Cov}\left(\int_{\mathbb{R}} f d N, \int_{\mathbb{R}} g d N\right)=\lambda_{0} \sum_{k=-\infty}^{\infty} c(|k|) \int_{\mathbb{R}} \mathbb{E}\left(f(s) g\left(s+T_{k}\right)\right) d s
$$

where $c(k)=\mathbb{E}(K-k+1)_{+}$.

(c) Let $f, g: \mathbb{R} \times \mathbb{R}_{+} \rightarrow \mathbb{R}_{+}$be measurable and $\mathbb{E}|Z|<\infty$. Then

$$
\begin{aligned}
\operatorname{Cov} & \left(\int_{\mathbb{R} \times \mathbb{R}_{+}} f d N^{(M)}, \int_{\mathbb{R} \times \mathbb{R}_{+}} g d N^{(M)}\right) \\
= & \lambda_{0} \sum_{\substack{k=-\infty \\
k \neq 0}}^{\infty} c(|k|) \int_{\mathbb{R} \times \mathbb{R}_{+}^{2}} \mathbb{E}\left(f\left(s, z_{1}\right) g\left(s+T_{k}, z_{2}\right)\right) F_{Z}\left(d z_{1}\right) F_{Z}\left(d z_{2}\right) d s \\
& +\lambda \int_{\mathbb{R}_{\mathbb{R}_{+}}} f(s, z) g(s, z) F_{Z}(d z) d s .
\end{aligned}
$$

(d) Let $\mathbb{E}|Z|<\infty$ and $h_{1}, h_{2}, h_{3} \geq 0, h_{1} \leq h_{2}<h_{3}$. Then

$$
\operatorname{Cov}\left(A\left(h_{1}\right), A\left(h_{3}\right)-A\left(h_{2}\right)\right)=\int_{h_{2}-h_{1}}^{h_{3}}\left(\min \left(h_{1}, h_{3}-x\right)-\left(h_{2}-x\right)_{+}\right) g(x) d x
$$


where

$$
\begin{aligned}
g(t)= & \lambda\left[\mathbb{E}\left(Z_{1}-t\right)_{+}-\mathbb{E}\left(Z_{1} \wedge\left(Z_{2}-t\right)_{+}\right)\right] \\
& +\int_{\mathbb{R}^{2}} \mathbf{1}_{\left\{s_{1} \leq 0, s_{2} \leq t\right\}} \bar{F}_{Z}\left(-s_{1}\right) \bar{F}_{Z}\left(t-s_{2}\right) \gamma_{2}^{(N)}\left(d s_{1}, d s_{2}\right)
\end{aligned}
$$

and

$$
\gamma_{2}^{N}(A \times B)=\lambda_{0} \sum_{k=-\infty}^{\infty} c(|k|) \int_{A} \mathbb{P}\left(s+T_{k} \in B\right) \operatorname{Leb}(d s) \quad \text { for } A, B \in \mathcal{B}\left(\mathbb{R}_{+}\right)
$$

is the covariance measure of $N$.

A conclusion of Lemma 2.1 (a) and (2.5) is that $A(t)$ has a finite mean under the assumption that $Z$ has a finite mean and

$$
\mathbb{E}(A(t))=\lambda t \mathbb{E}(Z)
$$

(cf. Mikosch and Samorodnitsky [26], Lemma 2.2).

\subsection{Model assumptions}

In this paper the within-cluster interarrival times and the cluster sizes are assumed to be heavytailed which is modeled by regularly varying tail distribution functions. For a positive measurable function $f$ we write $f \in \mathcal{R}_{\kappa}$ for $\kappa \in \mathbb{R}$ ( $f$ is regularly varying with index $\left.\kappa\right)$ if

$$
\lim _{x \rightarrow \infty} \frac{f(t x)}{f(x)}=t^{\kappa} \quad \forall t>0 .
$$

More details on regular variation can be found, e.g., in Bingham et al. [6] and Resnick [30].

\section{Assumption A}

(a) The interarrival distribution function $F_{X}$ satisfies

$$
\bar{F}_{X} \in \mathcal{R}_{-1 / \beta} \quad \text { with } \beta>1 \text {. }
$$

(b) The cluster size distribution function $F_{K}$ satisfies

$$
\bar{F}_{K} \in \mathcal{R}_{-\alpha} \quad \text { with } 1<\alpha<\min (2, \beta) .
$$

Notice that Assumption A (a) assures that the within-cluster interarrival times have infinite mean; it also makes the arrival process sufficiently irregular for our results. Assumption $A$ (b) makes sure that the file sizes transmitted within each cluster have infinite variance.

\section{Assumption B}

Assume that either of the following conditions is satisfied:

(a) $\beta<2$ and

$$
\limsup _{x \rightarrow \infty} x \frac{\bar{F}_{X}(x)-\bar{F}_{X}(x+1)}{\bar{F}_{X}(x)}<\infty .
$$


(b) $F$ is arithmetic, with step size $\Delta>0$, and

$$
\limsup _{n \geq 0} n \frac{F_{X}(\{n \Delta\})}{\bar{F}_{X}(n \Delta)}<\infty .
$$

Remark 2.2 We need the technical Assumption $B$ above to apply a local renewal theorem in Lemma 2.3 (d) below. In fact, if the local renewal theorem is known to hold, then Assumption $B$ is unnecessary. We conjecture that the local renewal theorem holds under (2.8) for any $\beta>1$, regardless of whether or not $F$ is arithmetic.

\section{Assumption C}

The marks $\left(\mathcal{Z}_{l}\right)$ satisfy one of the following conditions:

(a) $\mathbb{E}|Z|^{2}<\infty$. Then we define $\gamma:=2$.

(b) $\bar{F}_{Z} \in \mathcal{R}_{-\gamma}$ for some $1<\gamma<2$.

Two conclusions from this assumption are important for our study. First, we have by Markov's inequality (in case (a)) and Karamata's Theorem (in case (b)) (cf. Resnick [30], Theorem 2.1), respectively, the following upper bound of the tail of $F_{Z}$ :

$$
\mathbb{P}(Z>x) \leq C x^{-\gamma_{1}} \quad \text { for } x \geq 1,
$$

where $\gamma_{1}=2$ if $\gamma=2$, and $\gamma_{1}<\gamma$ if $1<\gamma<2$, respectively. In this paper $\gamma_{1}$ will be chosen sufficiently close to $\gamma$. Second, if we define

$$
\begin{array}{lll}
a(n):=n^{1 / 2} & \text { if } \gamma=2, \text { and } \\
a(n):=\bar{F}_{Z}^{\leftarrow}\left(n^{-1}\right) & \text { if } 1<\gamma<2,
\end{array}
$$

then by Resnick [30], Theorem 3.3 and Corollary 7.1 we have the central limit theorem

$$
\left(\frac{1}{a(n)} \sum_{l=1}^{\lfloor n t\rfloor}\left[\mathcal{Z}_{l}-\mathbb{E}(Z)\right]\right)_{t \geq 0} \Longrightarrow\left(S_{\gamma}(t)\right)_{t \geq 0} \quad \text { as } n \rightarrow \infty \text { in }\left(\mathbb{D}[0, \infty), J_{1}\right),
$$

where $\left(S_{\gamma}(t)\right)_{t \geq 0}$ is a $\gamma$-stable Lévy process and for $\gamma=2$ the limit process is a Brownian motion. The space $\mathbb{D}[0,1](\mathbb{D}[0, \infty)$, respectively) denotes the space of right continuous functions on $[0,1]$ $([0, \infty)$, respectively) with left limits (shortly: càdlàg = continue à droite, limitée à gauche). The symbol $J_{1}$ reflects that $\mathbb{D}[0,1]$ is provided with the Skorokhod $J_{1}$ topology. For completeness, $\mathbb{C}[0,1]$ denotes the space of continuous functions on $[0,1]$ which we provide with the uniform metric; see Billingsley [5].

Throughout the paper we use the following notation:

$$
\begin{aligned}
H & =\frac{2+\beta-\alpha}{2 \beta}, \\
c(k) & =\mathbb{E}(K-k+1)_{+}, \quad k \in \mathbb{N}_{0}, \\
G(t) & =\sum_{k=0}^{\infty} c(k) \mathbb{P}\left(T_{k} \leq t\right), \quad t \geq 0, \\
V(t) & =D(\alpha, \beta) t^{-1}(\alpha-1)^{-1} \bar{F}_{X}(t)^{-2} \mathbb{P}\left(K>\bar{F}_{X}(t)^{-1}\right), \quad t \geq 0,
\end{aligned}
$$


where

$$
D(\alpha, \beta)=\frac{\Gamma\left(1-\beta^{-1}\right)^{2-\alpha} \Gamma(3-\alpha)}{\beta \Gamma(2 H)} .
$$

Here, $\Gamma$ denotes the $\Gamma$-function. We further use that under Assumption $A$ the inequalities

$$
\frac{1}{2}<H<1 \quad \text { and } \quad 1<2-H<H^{-1}<3-2 H<2
$$

hold. The next Lemma is crucial for the proof of our results.

Lemma 2.3 Let Assumption A hold. Then we have:

(a) $c(k) \sim(\alpha-1)^{-1} k \bar{F}_{K}(k) \in \mathcal{R}_{1-\alpha}$ as $k \rightarrow \infty$.

(b) $V(t) \in \mathcal{R}_{2 H-2}$ as $t \rightarrow \infty$, where $2 H-2 \in(-1,0)$.

(c) $G(t) \sim(2 H-1)^{-1} t V(t) \in \mathcal{R}_{2 H-1}$ as $t \rightarrow \infty$, where $2 H-1 \in(0,1)$.

(d) Let additionally Assumption $B$ hold. Then $G(t)-G(t-1) \sim V(t) \in \mathcal{R}_{2 H-2}$ as $t \rightarrow \infty$, where $2 H-2 \in(-1,0)$.

\section{Asymptotic behavior of the variance}

We start with the investigation of the asymptotic behavior of the variance of $N$ and $A$. These results are essential to the understanding of the asymptotic behavior of these processes in Section 4 and 5 .

Proposition 3.1 Let Assumption A hold. Then $\operatorname{Var}(N(0, \cdot]) \in \mathcal{R}_{2 H}$ and

$$
\operatorname{Var}(N(0, t]) \sim \frac{1}{H(2 H-1)} \lambda_{0} t^{2} V(t) \quad \text { as } t \rightarrow \infty .
$$

Proposition 3.2 Let Assumption A, B and $C$ hold.

(a) If $\gamma>3-2 H$ then $\operatorname{Var}(A(\cdot)) \in \mathcal{R}_{2 H}$, where $2 H \in(1,2)$, and

$$
\operatorname{Var}(A(t)) \sim \frac{(\mathbb{E}(Z))^{2}}{H(2 H-1)} \lambda_{0} t^{2} V(t) \sim(\mathbb{E}(Z))^{2} \operatorname{Var}(N(0, t]) \quad \text { as } t \rightarrow \infty .
$$

(b) If $\gamma<3-2 H$ then $\operatorname{Var}(A(\cdot)) \in \mathcal{R}_{3-\gamma}$, where $3-\gamma \in(1,2)$, and

$$
\operatorname{Var}(A(t)) \sim \frac{2 \lambda}{(2-\gamma)(3-\gamma)(\gamma-1)} t^{3} \bar{F}_{Z}(t) \quad \text { as } t \rightarrow \infty .
$$

\section{Remark 3.3}

(a) If $\gamma>3-2 H$ the asymptotic behavior of the variance is completely determined by the point process $N$. The file sizes $Z$ have no influence on the rate of increase of the variance. 
(b) In contrast, if $\gamma<3-2 H$, the rate of increase of the variance comes only from the file sizes. If we have an infinite source Poisson model with $\bar{F}_{Z} \in \mathcal{R}_{-\gamma}$ for some $1<\gamma<2$ then we have the same asymptotic behavior of the variance (cf. Mikosch and Samorodnitsky [26], p. 895).

Similarly we derive now the asymptotic behavior of the covariance functions which show the long range dependence of $N$ and $A$.

Theorem 3.4 Let Assumption A, B and C hold. Define for $h \geq 1$,

$$
\begin{aligned}
& \gamma_{N}(h):=\operatorname{Cov}(N(0,1], N(h, h+1]), \\
& \gamma_{A}(h):=\operatorname{Cov}(A(1), A(h+1)-A(h)) .
\end{aligned}
$$

Then $\gamma_{N} \in \mathcal{R}_{2 H-2}$, where $2 H-2 \in(-1,0)$, and

$$
\gamma_{N}(h) \sim \lambda_{0} V(h) \quad \text { as } h \rightarrow \infty .
$$

(a) If $\gamma>3-2 H$ then $\gamma_{A} \in \mathcal{R}_{2 H-2}$, where $2 H-2 \in(-1,0)$, and

$$
\gamma_{A}(h) \sim(\mathbb{E}(Z))^{2} \gamma_{N}(h) \sim(\mathbb{E}(Z))^{2} \lambda_{0} V(h) \quad \text { as } h \rightarrow \infty .
$$

(b) If $\gamma<3-2 H$ then $\gamma_{A} \in \mathcal{R}_{1-\gamma}$, where $1-\gamma \in(-1,0)$, and

$$
\gamma_{A}(h) \sim \frac{\lambda}{\gamma-1} h \bar{F}_{Z}(h) \quad \text { as } h \rightarrow \infty .
$$

\section{Asymptotic behavior of the number of packets}

An interesting question itself is the behavior of the arrival process of data files modeled by the point process $N$. On the other hand, we require that result also for the derivation of the asymptotic behavior of the cumulative input to the server.

Theorem 4.1 Let Assumption $A$ and $B$ hold, and let $\left(M_{n}\right)$ be a sequence of positive constants such that $M_{n} \uparrow \infty$ as $n \rightarrow \infty$.

(a) Then

$$
\left(\frac{N\left(0, M_{n} t\right]-\lambda M_{n} t}{\sqrt{\operatorname{Var}\left(N\left(0, M_{n}\right]\right)}}\right)_{t \geq 0} \Longrightarrow\left(B_{H}(t)\right)_{t \geq 0} \quad \text { as } n \rightarrow \infty \text { in } \mathbb{C}[0,1],
$$

where $\left(B_{H}(t)\right)_{t \geq 0}$ is a standard fractional Brownian motion with Hurst index $H$ given in (2.12).

(b) Let $\left(N_{i}\right)$ be iid copies of $N$. Then

$$
\frac{1}{\sqrt{n \operatorname{Var}\left(N\left(0, M_{n}\right]\right)}} \sum_{i=1}^{n}\left[N_{i}\left(0, M_{n} t\right]-\lambda M_{n} t\right] \Longrightarrow\left(B_{H}(t)\right)_{t \geq 0} \quad \text { as } n \rightarrow \infty \text { in } \mathbb{C}[0,1] \text {. }
$$

Astonishingly in contrast to the results in Faÿ et al. [14] the limit results in (a) and (b) do not depend on the growth rate of $\left(M_{n}\right)$. 


\section{$5 \quad$ Asymptotic behavior of the input process}

Let us start now with the asymptotic behavior of a mean corrected individual input process $A$. Afterwards we will investigate the cumulative input to the server.

Theorem 5.1 Let Assumption $A, B$ and $C$ hold and let $\left(M_{n}\right)$ be a sequence of positive constants such that $M_{n} \uparrow \infty$ as $n \rightarrow \infty$.

(a) Let $\gamma>H^{-1}$. Then

$$
\left(\frac{A\left(M_{n} t\right)-\lambda M_{n} t \mathbb{E}(Z)}{\sqrt{\operatorname{Var}\left(N\left(0, M_{n}\right]\right)}}\right)_{t \geq 0} \stackrel{F D D}{\longrightarrow}\left(\mathbb{E}(Z) B_{H}(t)\right)_{t \geq 0} \quad \text { as } n \rightarrow \infty,
$$

where $\left(B_{H}(t)\right)_{t \geq 0}$ is a standard fractional Brownian motion with Hurst index $H$ given in (2.12). In particular, if $\gamma>3-2 H$ then

$$
\left(\frac{A\left(M_{n} t\right)-\lambda M_{n} t \mathbb{E}(Z)}{\sqrt{\operatorname{Var}\left(A\left(M_{n}\right)\right)}}\right)_{t \geq 0} \Longrightarrow\left(B_{H}(t)\right)_{t \geq 0} \quad \text { as } n \rightarrow \infty \text { in } \mathbb{C}[0,1] \text {. }
$$

(b) Let $\gamma<H^{-1}$. Then

$$
\left(\frac{A\left(M_{n} t\right)-\lambda M_{n} t \mathbb{E}(Z)}{a\left(M_{n}\right)}\right)_{t \geq 0} \stackrel{F D D}{\Longrightarrow}\left(\lambda^{\frac{1}{\gamma}} S_{\gamma}(t)\right)_{t \geq 0} \quad \text { as } n \rightarrow \infty
$$

where $\left(S_{\gamma}(t)\right)_{t \geq 0}$ is the $\gamma$-stable Lévy motion given in (2.11).

\section{Remark 5.2}

(i) In the case $H^{-1}<\gamma<3-2 H$ we have only stated the convergence of the finite dimensional distributions. To show the tightness in $\mathbb{C}[0,1]$ we can not use the classical arguments of Billingsley [5] since the process does not converge in $\mathcal{L}_{2}$. However, we conjecture that the convergence holds in $\mathbb{C}[0,1]$ by proving the $\mathcal{L}_{\delta}$ convergence for some $\delta \in\left(H^{-1}, \gamma\right)$. See also Remark 6.5 in Section 6.4.

(ii) Since the left hand side in (b) has continuous sample paths but the right hand side has jumps, the convergence can not hold in $\left(\mathbb{D}[0,1], J_{1}\right)$; cf. Whitt $[33,34]$. However, we conjecture that with some technical effort we can extend the result to convergence in $\left(\mathbb{D}[0,1], M_{1}\right)$ as in Resnick and Van den Berg [29]. Analog statements hold for further results presented in this paper; cf. Theorem 5.5 and Theorem 5.6.

For the asymptotic behavior of the mean corrected cumulative input process we have to distinguish again different cases depending on the heaviness of the file sizes. The reason for these cases is that we have on the one hand, the two different cases for the asymptotic behavior of the variance $(\gamma>3-2 H, \gamma<3-2 H)$ and on the other hand, the two different cases for the asymptotic behavior of one mean corrected input process $\left(\gamma>H^{-1}, \gamma<H^{-1}\right)$. These cases result also in different cases for the asymptotic behavior of the mean corrected cumulative input process; $3-2 H<\gamma \leq 2, H^{-1}<\gamma<3-2 H$ and $1<\gamma<H^{-1}$. Recall that $1<H^{-1}<3-2 H<2$. 


\section{$5.13-2 H<\gamma \leq 2$}

Theorem 5.3 Let Assumption $A, B$ and $C$ hold. Further, let $\left(M_{n}\right)$ be a sequence of positive constants such that $M_{n} \uparrow \infty$ as $n \rightarrow \infty$, and either

(a) $\liminf \operatorname{in}_{n \rightarrow \infty} n^{-\frac{1}{\gamma-1}+\eta} M_{n}>0$ for every $\eta>0$, or

(b) $\lim _{n \rightarrow \infty} n^{-\frac{1}{\gamma-1}+\eta} M_{n}=0$ for some $\eta>0$.

Further, let $\left(S_{n, M_{n}}(t)\right)_{t \geq 0}$ be given as in (1.1) and suppose $\gamma>3-2 H$. Then

$$
\left(\left[n \operatorname{Var}\left(A\left(M_{n}\right)\right)\right]^{-1 / 2} S_{n, M_{n}}(t)\right)_{t \geq 0} \Longrightarrow\left(B_{H}(t)\right)_{t \geq 0} \quad \text { as } n \rightarrow \infty \text { in } \mathbb{C}[0,1] \text {. }
$$

In particular, if

$$
\lim _{n \rightarrow \infty} n^{-\frac{1}{\gamma-1}} M_{n}=C \in(0, \infty]
$$

holds, then (a) is valid. The conditions (a) and (b) imply a kind of continuity condition on $\left(M_{n}\right)$ which is natural. However, if $\mathbb{E}\left(Z^{2}\right)<\infty$ we can neglect the continuity condition; see Theorem 5.4 below.

In the classical results as the ON/OFF and the infinite source Poisson model with heavytailed file sizes we have contrary to Theorem 5.3 to distinguish between fast and slow growth rates of $\left(M_{n}\right)$. In the slow growth case we obtain the convergence to a $\gamma$-stable Lévy motion. Hence, our general Poisson cluster model shows that this is not necessarily the case if we have heavy-tailed file sizes.

Theorem 5.4 Let Assumption $A, B$ and $C$ hold. Further, let $\left(M_{n}\right)$ be a sequence of positive constants such that $M_{n} \uparrow \infty$ as $n \rightarrow \infty$, and let $\left(S_{n, M_{n}}(t)\right)_{t \geq 0}$ be given as in (1.1). Suppose that $\mathbb{E}\left(Z^{2}\right)<\infty$. Then

$$
\left(\left[n \operatorname{Var}\left(A\left(M_{n}\right)\right)\right]^{-1 / 2} S_{n, M_{n}}(t)\right)_{t \geq 0} \Longrightarrow\left(B_{H}(t)\right)_{t \geq 0} \quad \text { as } n \rightarrow \infty \text { in } \mathbb{C}[0,1] \text {. }
$$

\section{$5.2 \quad H^{-1}<\gamma<3-2 H$}

Theorem 5.5 Let Assumption A, B and $C$ hold and let $H^{-1}<\gamma<3-2 H$. Further, let $\left(M_{n}\right)$ be a sequence of positive constants such that $M_{n} \uparrow \infty$ as $n \rightarrow \infty$, and $\left(S_{n, M_{n}}(t)\right)_{t \geq 0}$ be given as in (1.1).

(a) Let $\left(M_{n}\right)$ satisfies the fast growth condition

$$
\lim _{n \rightarrow \infty} n^{-\frac{1}{\gamma-1}+\eta} M_{n}=0
$$

for some $\eta>0$. Then

$$
\left(\left[n \operatorname{Var}\left(A\left(M_{n}\right)\right)\right]^{-1 / 2} S_{n, M_{n}}(t)\right)_{t \geq 0} \Longrightarrow\left(B_{\frac{3-\gamma}{2}}(t)\right)_{t \geq 0} \quad \text { as } n \rightarrow \infty \text { in } \mathbb{C}[0,1] .
$$


(b) Let $\left(M_{n}\right)$ satisfies the intermediate growth condition

$$
\lim _{n \rightarrow \infty} n^{\frac{1}{\gamma-1}+\eta} M_{n}^{-1}=0
$$

and

$$
\lim _{n \rightarrow \infty} n^{\frac{2-\gamma}{2 H \gamma-2}-\eta} M_{n}^{-1}=\infty
$$

for some $\eta>0$. Then

$$
\left(a\left(n M_{n}\right)^{-1} S_{n, M_{n}}(t)\right)_{t \geq 0} \stackrel{F D D}{\Longrightarrow}\left(\lambda^{\frac{1}{\gamma}} S_{\gamma}(t)\right)_{t \geq 0} \quad \text { as } n \rightarrow \infty .
$$

(c) Let $\left(M_{n}\right)$ satisfies the slow growth condition

$$
\lim _{n \rightarrow \infty} n^{\frac{2-\gamma}{2 H \gamma}+\eta} M_{n}^{-1}=0
$$

for some $\eta>0$. Then

$$
\left(\left[n \operatorname{Var}\left(N\left(0, M_{n}\right]\right)\right]^{-1 / 2} S_{n, M_{n}}(t)\right)_{t \geq 0} \stackrel{F D D}{\longrightarrow}\left(\mathbb{E}(Z) B_{H}(t)\right)_{t \geq 0} \quad \text { as } n \rightarrow \infty .
$$

\section{$5.31<\gamma<H^{-1}$}

Theorem 5.6 Let Assumption $A, B$ and $C$ hold and let $1<\gamma<H^{-1}$. Further, let $\left(M_{n}\right)$ be a sequence of positive constants such that $M_{n} \uparrow \infty$ as $n \rightarrow \infty$, and $\left(S_{n, M_{n}}(t)\right)_{t \geq 0}$ be given as in (1.1).

(a) Let $\left(M_{n}\right)$ satisfies the fast growth condition

$$
\lim _{n \rightarrow \infty} n^{-\frac{1}{\gamma-1}+\eta} M_{n}=0
$$

for some $\eta>0$. Then

$$
\left(\left[n \operatorname{Var}\left(A\left(M_{n}\right)\right)\right]^{-1 / 2} S_{n, M_{n}}(t)\right)_{t \geq 0} \Longrightarrow\left(B_{\frac{3-\gamma}{2}}(t)\right)_{t \geq 0} \quad \text { as } n \rightarrow \infty \text { in } \mathbb{C}[0,1] .
$$

(b) Let $\left(M_{n}\right)$ satisfies the slow growth condition

$$
\lim _{n \rightarrow \infty} n^{\frac{1}{\gamma-1}+\eta} M_{n}^{-1}=0
$$

for some $\eta>0$. Then

$$
\left(a\left(n M_{n}\right)^{-1} S_{n, M_{n}}(t)\right)_{t \geq 0} \stackrel{F D D}{\longrightarrow}\left(\lambda^{\frac{1}{\gamma}} S_{\gamma}(t)\right)_{t \geq 0} \quad \text { as } n \rightarrow \infty .
$$

Remark 5.7 For the ON/OFF and the infinite source Poisson model with heavy-tailed file sizes the growth rates of $\left(M_{n}\right)$ can be equivalently described by the asymptotics of the covariance function $\gamma_{A}$. Here, this is only possible for $1<\gamma<H^{-1}$. 


\section{Proofs}

\subsection{Proofs of Section 2}

\section{Proof of Lemma 2.1}

(a) Consider the marked point process

$$
N^{*}=\sum_{k=-\infty}^{\infty} \varepsilon_{\left(\Gamma_{k}, K_{k},\left(X_{j, k}\right)_{j \in \mathbb{N}},\left(Z_{j, k}\right)_{j \in \mathbb{N}_{0}}\right)} \quad \text { in } \quad \mathbb{R} \times \mathbb{N}_{0} \times \mathbb{R}_{+}^{\mathbb{N}} \times \mathbb{R}_{+}^{\mathbb{N}_{0}},
$$

which is a Poisson random measure with intensity measure

$$
m^{*}=\left(\lambda_{0} \times \mathrm{Leb}\right) \times F_{K} \times \prod_{j=1}^{\infty} F_{X} \prod_{l=0}^{\infty} F_{Z} .
$$

Let $f^{*}: \mathbb{R} \times \mathbb{N}_{0} \times \mathbb{R}_{+}^{\mathbb{N}} \times \mathbb{R}_{+}^{\mathbb{N}_{0}} \rightarrow \mathbb{R}$ be defined as

$$
f^{*}\left(s, k,\left(x_{j}\right),\left(z_{j}\right)\right)=\sum_{l=0}^{k} f\left(s+\sum_{j=1}^{l} x_{j}, z_{l}\right) .
$$

Then $\int_{\mathbb{R} \times \mathbb{R}_{+}} f d N^{(M)}=\int_{\mathbb{R} \times \mathbb{N}_{0} \times \mathbb{R}_{+}^{\mathbb{N}} \times \mathbb{R}_{+}^{\mathbb{N}_{0}}} f^{*} d N^{*}$. Thus, by Campbell's formula (cf. Baccelli and Brémaud [2], p. 18)

$$
\begin{aligned}
& \mathbb{E}\left(\int_{\mathbb{R} \times \mathbb{R}_{+}} f d N^{(M)}\right) \\
& \quad=\mathbb{E}\left(\int_{\mathbb{R} \times \mathbb{N}_{0} \times \mathbb{R}_{+}^{\mathbb{N}} \times \mathbb{R}_{+}^{\mathbb{N}_{0}}} f^{*} d N^{*}\right)=\int_{\mathbb{R} \times \mathbb{N}_{0} \times \mathbb{R}_{+}^{\mathbb{N}} \times \mathbb{R}_{+}^{\mathbb{N}_{0}}} f^{*} d m^{*} \\
& \quad=\lambda_{0} \int_{\mathbb{R} \times \mathbb{N}_{0} \times \mathbb{R}_{+}^{\mathbb{N}} \times \mathbb{R}_{+}^{\mathbb{N}_{0}}} \sum_{l=0}^{k} f\left(s+\sum_{j=1}^{l} x_{j}, z_{l}\right) \prod_{l=0}^{\infty} F_{Z}\left(d z_{l}\right) \prod_{j=1}^{\infty} F_{X}\left(d x_{j}\right) F_{K}(d k) d s \\
& \quad=\lambda_{0}(\mathbb{E}(K)+1) \int_{\mathbb{R} \times \mathbb{R}_{+}} f(s, z) F_{Z}(d z) d s .
\end{aligned}
$$

(b) The covariance measure $\gamma_{2}^{N}$ of $N$ is given by

$$
\gamma_{2}^{(N)}(A \times B)=\operatorname{Cov}(N(A), N(B))=\lambda_{0} \int_{\mathbb{R}} \widehat{\gamma}^{*}((s+A) \times(s+B)) d s
$$

where

$$
\widehat{\gamma}^{*}(A \times B)=\mathbb{E}\left(\sum_{k=0}^{K} \sum_{m=0}^{K} \mathbf{1}_{\left\{T_{k} \in A, T_{m} \in B\right\}}\right) \quad \text { for } A, B \in \mathcal{B}(\mathbb{R}) ;
$$

see Faÿ et al. [14], Proposition 2.3. Let

$$
\begin{aligned}
\gamma^{*}(A) & :=\mathbb{E}\left(\sum_{k=0}^{K} \sum_{m=0}^{K} \mathbf{1}_{\left\{T_{k}-T_{m} \in A\right\}}\right) \\
& =\mathbb{E}\left(\sum_{k=0}^{K}(K-k+1) \mathbf{1}_{\left\{T_{k} \in A\right\}}\right)+\mathbb{E}\left(\sum_{k=1}^{K}(K-k+1) \mathbf{1}_{\left\{-T_{k} \in A\right\}}\right) \\
& =\sum_{k=1}^{\infty} c(k)\left[\mathbb{P}\left(T_{k} \in A\right)+\mathbb{P}\left(-T_{k} \in A\right)\right]+c(0) \mathbf{1}_{\{0 \in A\}} .
\end{aligned}
$$


Then by Campbell's formula

$$
\operatorname{Cov}\left(\int_{\mathbb{R}} f d N, \int_{\mathbb{R}} g d N\right)=\int_{\mathbb{R}^{2}} f\left(s_{1}\right) g\left(s_{2}\right) \gamma_{2}^{(N)}\left(d s_{1}, d s_{2}\right)=\lambda_{0} \int_{\mathbb{R}^{2}} f(s) g(s+v) d s \gamma^{*}(d v)
$$

for measurable functions $f, g: \mathbb{R} \rightarrow \mathbb{R}$. In the case $f, g$ positive this can be rewritten as

$$
\operatorname{Cov}\left(\int_{\mathbb{R}} f d N, \int_{\mathbb{R}} g d N\right)=\lambda_{0} \sum_{k=-\infty}^{\infty} c(|k|) \int_{\mathbb{R}} \mathbb{E}\left(f(s) g\left(s+T_{k}\right)\right) d s
$$

(c) Finally, the covariance measure $\gamma_{2}^{(M)}$ of the marked point process $N^{(M)}$ is given by

$$
\gamma_{2}^{(M)}\left(d s_{1}, d s_{2}, d z_{1}, d z_{2}\right)=\gamma_{2}^{(N)}\left(d s_{1}, d s_{2}\right) F_{Z}\left(d z_{1}\right) F_{Z}\left(d z_{2}\right)+\gamma^{(d)}\left(d s_{1}, d s_{2}, d z_{1}, d z_{2}\right)
$$

where $\gamma^{(d)}$ is the signed diagonal measure

$$
\gamma^{(d)}\left(A_{1} \times A_{2} \times B_{1} \times B_{2}\right)=\lambda \operatorname{Leb}\left(A_{1} \cap A_{2}\right)\left[F_{Z}\left(B_{1} \cap B_{2}\right)-F_{Z}\left(B_{1}\right) F_{Z}\left(B_{2}\right)\right]
$$

for $A_{1}, A_{2} \in \mathcal{B}(\mathbb{R}), B_{1}, B_{2} \in \mathcal{B}\left(\mathbb{R}_{+}\right)$. This means

$$
\operatorname{Cov}\left(N^{(M)}\left(A_{1} \times B_{1}\right), N^{(M)}\left(A_{2} \times B_{2}\right)\right)=\gamma_{2}^{(M)}\left(A_{1} \times A_{2} \times B_{1} \times B_{2}\right) .
$$

For more details we refer to Mikosch and Samorodnitsky [26], p. 895. Again Campbell's formula gives

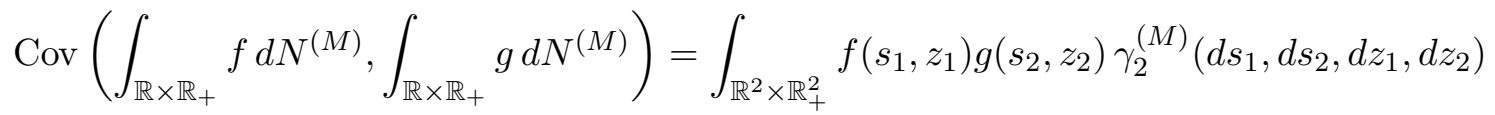

for measurable functions $f, g: \mathbb{R} \times \mathbb{R}_{+} \rightarrow \mathbb{R}$. If $f, g$ are positive the last equation reduces by (6.3) and (6.4) to

$$
\begin{aligned}
& \operatorname{Cov}\left(\int_{\mathbb{R} \times \mathbb{R}_{+}} f d N^{(M)}, \int_{\mathbb{R} \times \mathbb{R}_{+}} g d N^{(M)}\right) \\
& =\lambda_{0} \sum_{\substack{k=-\infty \\
k \neq 0}}^{\infty} c(|k|) \int_{\mathbb{R} \times \mathbb{R}_{+}^{2}} \mathbb{E}\left(f\left(s, z_{1}\right) g\left(s+T_{k}, z_{2}\right)\right) F_{Z}\left(d z_{1}\right) F_{Z}\left(d z_{2}\right) d s \\
& +\lambda \int_{\mathbb{R} \times \mathbb{R}_{+}} f(s, z) g(s, z) F_{Z}(d z) d s \\
& =: V_{1}(t)+V_{2}(t) \text {. }
\end{aligned}
$$

(d) Let us define

$$
f(s, z):=\int_{\left[0, h_{1}\right]} \mathbf{1}_{\{s<x<s+z\}} d x \quad \text { and } \quad \widetilde{f}(s, z):=\int_{\left[h_{2}, h_{3}\right]} \mathbf{1}_{\{s<x<s+z\}} d x
$$

for $s \in \mathbb{R}, z \geq 0$. Then

$$
A\left(h_{1}\right)=\int_{{\mathbb{R} \times \mathbb{R}_{+}}} f d N^{(M)} \quad \text { and } \quad A\left(h_{3}\right)-A\left(h_{2}\right)=\int_{{\mathbb{R} \times \mathbb{R}_{+}}} \tilde{f} d N^{(M)} .
$$


Thus, a conclusion of (6.5) is

$$
\begin{aligned}
\operatorname{Cov} & \left(A\left(h_{1}\right), A\left(h_{3}\right)-A\left(h_{2}\right)\right) \\
& =\int_{\mathbb{R}^{2} \times \mathbb{R}_{+}^{2}} f\left(s_{1}, z_{1}\right) \tilde{f}\left(s_{2}, z_{2}\right) \gamma_{2}^{(M)}\left(d s_{1}, d s_{2}, d z_{1}, d z_{2}\right) \\
& =\int_{\mathbb{R}^{2} \times \mathbb{R}_{+}^{2}} \int_{\left[0, h_{1}\right]} \mathbf{1}_{\left\{s_{1}<x<s_{1}+z_{1}\right\}} d x \int_{\left[h_{2}, h_{3}\right]} \mathbf{1}_{\left\{s_{2}<y<s_{2}+z_{2}\right\}} d y \gamma_{2}^{(M)}\left(d s_{1}, d s_{2}, d z_{1}, d z_{2}\right) \\
& =\int_{\left[0, h_{1}\right] \times\left[h_{2}, h_{3}\right]} \int_{\mathbb{R}^{2} \times \mathbb{R}_{+}^{2}} \mathbf{1}_{\left\{s_{1}<x<s_{1}+z_{1}, s_{2}<y<s_{2}+z_{2}\right\}} \gamma_{2}^{(M)}\left(d s_{1}, d s_{2}, d z_{1}, d z_{2}\right) d x d y .
\end{aligned}
$$

If we define

$$
g(x, y)=\int_{\mathbb{R}^{2} \times \mathbb{R}_{+}^{2}} \mathbf{1}_{\left\{s_{1}<x<s_{1}+z_{1}, s_{2}<y<s_{2}+z_{2}\right\}} \gamma_{2}^{(M)}\left(d s_{1}, d s_{2}, d z_{1}, d z_{2}\right),
$$

then

$$
\operatorname{Cov}\left(A\left(h_{1}\right), A\left(h_{3}\right)-A\left(h_{2}\right)\right)=\int_{\left[0, h_{1}\right] \times\left[h_{2}, h_{3}\right]} g(x, y) d x d y .
$$

Since $N^{(M)}$ is stationary, $g$ depends only on the difference $|y-x|$. Thus, let $g(v):=g(0, v)$. Then

$$
\begin{aligned}
\operatorname{Cov}\left(A\left(h_{1}\right), A\left(h_{3}\right)-A\left(h_{2}\right)\right) & =\int_{0}^{h_{1}} \int_{h_{2}-x}^{h_{3}-x} g(x, x+v) d v d x \\
& =\int_{0}^{h_{1}} \int_{h_{2}-x}^{h_{3}-x} g(v) d v d x \\
& =\int_{h_{2}-h_{1}}^{h_{3}}\left[\min \left(h_{1}, h_{3}-v\right)-\left(h_{2}-v\right)_{+}\right] g(v) d v .
\end{aligned}
$$

Furthermore, (6.3) and (6.4) give

$$
\begin{aligned}
g(v) & =\int_{\mathbb{R}^{2} \times \mathbb{R}_{+}^{2}} \mathbf{1}_{\left\{s_{1}<0<s_{1}+z_{1}, s_{2}<v<s_{2}+z_{2}\right\}} \gamma_{2}^{(M)}\left(d s_{1}, d s_{2}, d z_{1}, d z_{2}\right) \\
& =\lambda\left[\mathbb{E}\left(Z_{1}-v\right)_{+}-\mathbb{E}\left(Z_{1} \wedge\left(Z_{2}-v\right)_{+}\right)\right]+\int_{\mathbb{R}^{2}} \mathbf{1}_{\left\{s_{1} \leq 0, s_{2} \leq v\right\}} \bar{F}_{Z}\left(-s_{1}\right) \bar{F}_{Z}\left(v-s_{2}\right) \gamma_{2}^{(N)}\left(d s_{1}, d s_{2}\right) .
\end{aligned}
$$

Proof of Lemma 2.3. (a) follows from Karamata's theorem and (b) by the definition of $V$. (c) is a conclusion from Omey [27] and (d) from Anderson and Athreya [1], Theorem 2, if Assumption $B$ (a) holds and from Doney [12], Theorem 3, if Assumption B (b) holds. By Zolotarev [36], Theorem 3, the constants in [1] and [12] are equal. 


\subsection{Proofs of Section 3}

Proof of Proposition 3.1. If we insert $f=g=\mathbf{1}_{(0, t]}$ in Lemma 2.1 (b) we obtain

$$
\begin{aligned}
\operatorname{Var}(N(0, t]) & =\lambda_{0} \sum_{k=-\infty}^{\infty} c(|k|) \int_{0}^{t} \mathbb{P}\left(0<s+T_{k} \leq t\right) d s \\
& =\lambda t+2 \lambda_{0} \sum_{k=1}^{\infty} c(k) \int_{0}^{t} \mathbb{P}\left(T_{k} \leq v\right) d v \\
& =\lambda t+2 \lambda_{0} \int_{0}^{t} G(v) d v .
\end{aligned}
$$

Since $G \in \mathcal{R}_{2 H-1}, 2 H-1>0$, by Lemma 2.3 (c), we conclude with Karamata's Theorem,

$$
\operatorname{Var}(N(0, t]) \sim \frac{1}{H(2 H-1)} \lambda_{0} t^{2} V(t) \quad \text { as } t \rightarrow \infty .
$$

The proof of Proposition 3.2 is based on some auxiliary Lemmatas.

\section{Lemma 6.1}

(a) Let Assumption $A$ and $B$ hold, and let $h(z, t)=G(x+t)-G(x+t-z)$ for $x \geq 0$. Then there exist $t_{0}, C>0$ such that

$$
h(z, t) \leq C(z+1) V(t)
$$

for $x>0, z<t / 2, t \geq t_{0}$.

(b) Let Assumption $C$ hold, and let $h(t)=\mathbb{E}(Z-t)_{+}$. Then there exists a $C>0$ such that

$$
h(t) \leq C t^{1-\gamma_{1}}, \quad t \geq 1 .
$$

(c) Let Assumption $C$ hold, and let $h(t)=\int_{0}^{\infty} \bar{F}_{Z}(x) \bar{F}_{Z}(x+t) d x$. Then there exists a $C>0$ such that

$$
h(t) \leq C t^{-\gamma_{1}}, \quad t \geq 1 .
$$

(d) Let Assumption $A$ and $C$ hold, and let $h(t)=\sum_{k=1}^{\infty} c(k) \int_{0}^{\infty} \bar{F}_{Z}(x) \mathbb{P}\left(Z>x+t+T_{k}\right) d x$.

Then there exists a $C>0$ such that

$$
h(t) \leq C t^{2 H-\gamma_{1}-1}, \quad t \geq 1 .
$$

(e) Let Assumption A, B and C hold, and let

$$
h(t)=\int_{0}^{\infty} \bar{F}_{Z}(x) \sum_{k=1}^{\infty} c(k) \mathbb{P}\left(T_{k}<x+t \leq T_{k}+Z\right) d x .
$$

Then

$$
h(t) \sim(\mathbb{E}(Z))^{2} V(t) \quad \text { as } t \rightarrow \infty .
$$




\section{Proof.}

(a) By Lemma 2.3 we know that $G(t)-G(t-1) \sim V(t)$ as $t \rightarrow \infty$. Hence, there exist $C_{1}, t_{0}>0$ such that

$$
G(x+t)-G(x+t-1) \leq C_{1} V(x+t) \quad \text { for } x \geq 0, t \geq t_{0} .
$$

Let $z \leq t / 2$ and $t \geq 2 t_{0}$. Then

$$
\begin{aligned}
& G(x+t)-G(x+t-z) \leq G(x+t)-G(x+t-\lceil z\rceil) \\
& =\sum_{j=1}^{\lceil z\rceil}[G(x+t-j+1)-G(x+t-j)] \\
& \leq C_{1} \sum_{j=1}^{\lceil z\rceil} V(x+t-j+1) \\
& \leq C_{1}\lceil z\rceil \sup _{1 \leq j \leq t / 2} V(x+t-j+1) \\
& \leq C_{2}(z+1) V(t) \text {, }
\end{aligned}
$$

where we used $V(\cdot) \in \mathcal{R}_{2 H-2}$ and Theorem 1.5.3 of Bingham et al. [6].

(b) Inequality (2.10) gives

$$
\mathbb{E}(Z-t)_{+}=\int_{t}^{\infty} \mathbb{P}(Z>x) d x \leq C_{3} \int_{t}^{\infty} x^{-\gamma_{1}} d x=\frac{C_{3}}{\gamma_{1}-1} t^{1-\gamma_{1}} .
$$

(c) We have again by $(2.10)$

$$
h(t) \leq C_{4} \int_{0}^{\infty} \bar{F}_{Z}(x)(x+t)^{-\gamma_{1}} d x \leq C_{4} t^{-\gamma_{1}} \int_{0}^{\infty} \bar{F}_{Z}(x) d x=C_{4} \mathbb{E}(Z) t^{-\gamma_{1}} .
$$

(d) Note that

$$
\begin{aligned}
h(t) & =\int_{0}^{\infty} \bar{F}_{Z}(x) \sum_{k=1}^{\infty} c(k) \mathbb{P}\left(Z>x+t+T_{k}\right) d x \\
& =\int_{0}^{\infty} \bar{F}_{Z}(x) \int_{x+t}^{\infty} \sum_{k=1}^{\infty} c(k) \mathbb{P}\left(T_{k}<z-x-t\right) F_{Z}(d z) d x \\
& \leq \int_{0}^{\infty} \bar{F}_{Z}(x) \int_{x+t}^{\infty} G(z-x-t) F_{Z}(d z) d x .
\end{aligned}
$$

Since $G$ is non-decreasing we have

$$
h(t) \leq \int_{0}^{\infty} \bar{F}_{Z}(x) \int_{t}^{\infty} G(z) F_{Z}(d z) d x .
$$

Keep in mind that $G \in \mathcal{R}_{2 H-1}, \bar{F}_{Z} \in \mathcal{R}_{-\gamma}$, then Potter's Theorem and Karamata's Theorem give

$$
h(t) \leq C_{5} \mathbb{E}(Z) t^{2 H-\gamma_{1}-1}
$$


(e) We can write

$$
\begin{aligned}
h(t) & =\int_{0}^{\infty} \bar{F}_{Z}(x) \int_{0}^{\infty} \sum_{k=1}^{\infty} c(k) \mathbb{P}\left(x+t-z \leq T_{k}<x+t\right) F_{Z}(d z) d x \\
& =\int_{0}^{\infty} \bar{F}_{Z}(x) \int_{0}^{\infty}[G(x+t)-G(x+t-z)] F_{Z}(d z) d x \\
& =\int_{0}^{\infty} \bar{F}_{Z}(x)\left[\int_{0}^{t / 2}+\int_{t / 2}^{\infty}\right][G(x+t)-G(x+t-z)] F_{Z}(d z) d x \\
& =: h_{1}(t)+h_{2}(t) .
\end{aligned}
$$

Thus, we obtain by dominated convergence, (a) and Lemma 2.3 (d)

$$
\lim _{t \rightarrow \infty} \frac{h_{1}(t)}{V(t)}=\int_{0}^{\infty} \bar{F}_{Z}(x) \int_{0}^{\infty} z F_{Z}(d z) d x=(\mathbb{E}(Z))^{2} .
$$

Further,

$$
\begin{aligned}
h_{2}(t) & =\int_{0}^{\infty} \bar{F}_{Z}(x) \int_{t / 2}^{\infty}[G(x+t)-G(x+t-z)] F_{Z}(d z) d x \\
& \leq \int_{0}^{\infty} \bar{F}_{Z}(x) G(x+t) \bar{F}_{Z}(t / 2) d x \\
& =\bar{F}_{Z}(t / 2)\left[\int_{0}^{t}+\int_{t}^{\infty}\right] \bar{F}_{Z}(x) G(x+t) d x=: h_{21}(t)+h_{22}(t) .
\end{aligned}
$$

Then (2.10), Potter's Theorem and Lemma 2.3 (c) give for some $0<\epsilon<1$

$$
\begin{aligned}
h_{21}(t) & \leq C_{6} t^{-\gamma_{1}} \int_{0}^{t} \bar{F}_{Z}(x)(x+t)^{\frac{2-\alpha}{\beta}+\epsilon} d x \\
& =C_{6} t^{\frac{2-\alpha}{\beta}+\epsilon-\gamma_{1}} \int_{0}^{t} \bar{F}_{Z}(x)\left(\frac{x}{t}+1\right)^{\frac{2-\alpha}{\beta}+\epsilon} d x \\
& \leq C_{7} \mathbb{E}(Z) t^{\frac{2-\alpha}{\beta}+\epsilon-\gamma_{1}} .
\end{aligned}
$$

Next,

$$
\begin{aligned}
h_{22}(t) & \leq C_{8} t^{-\gamma_{1}} \int_{t}^{\infty} x^{-\gamma_{1}}(x+t)^{\frac{2-\alpha}{\beta}+\epsilon} d x \\
& =C_{8} t^{-\gamma_{1}} \int_{t}^{\infty} x^{\frac{2-\alpha}{\beta}+\epsilon-\gamma_{1}}\left(1+\frac{t}{x}\right)^{\frac{2-\alpha}{\beta}+\epsilon} d x \\
& \leq C_{9} t^{-\gamma_{1}} t^{\frac{2-\alpha}{\beta}+\epsilon-\gamma_{1}+1} .
\end{aligned}
$$

Finally, since $\frac{2-\alpha}{\beta}+\epsilon-\gamma_{1}=2 H-1+\epsilon-\gamma_{1} \leq 2 H-2$ for small $\epsilon$ we have

$$
h_{2}(t) \leq C_{10} t^{\frac{2-\alpha}{\beta}+\epsilon-\gamma_{1}}=o\left(h_{1}(t)\right) \quad \text { as } t \rightarrow \infty .
$$

Thus, (6.8)-(6.11) result in the proof of $(d)$.

Lemma 6.2 Let Assumption A, B and $C$ hold, and $g$ be defined as in (2.6). 
(a) If $\gamma>3-2 H$ then

$$
g(t) \sim \lambda_{0}(\mathbb{E}(Z))^{2} V(t) \in \mathcal{R}_{2 H-2} \quad \text { as } t \rightarrow \infty .
$$

(b) If $\gamma<3-2 H$ then

$$
g(t) \sim \frac{\lambda}{\gamma-1} t \bar{F}_{Z}(t) \in \mathcal{R}_{1-\gamma} \quad \text { as } t \rightarrow \infty
$$

Proof. We use the decomposition

$$
\begin{aligned}
g(t) & =\lambda\left[\mathbb{E}\left(Z_{1}-t\right)_{+}-\mathbb{E}\left(Z_{1} \wedge\left(Z_{2}-t\right)_{+}\right)\right]+\int_{\mathbb{R}^{2}} \mathbf{1}_{\left\{s_{1} \leq 0, s_{2} \leq t\right\}} \bar{F}_{Z}\left(-s_{1}\right) \bar{F}_{Z}\left(t-s_{2}\right) \gamma_{2}^{(N)}\left(d s_{1}, d s_{2}\right) \\
& =: g_{1}(t)+g_{2}(t) .
\end{aligned}
$$

From Lemma 6.1 (b) we conclude that

$$
g_{1}(t) \leq 2 \lambda \mathbb{E}(Z-t)_{+} \leq C t^{1-\gamma_{1}} .
$$

Further, we have (cf. (35) of Mikosch and Samorodnitsky [26])

$$
g_{2}(t)=\lambda h_{1}(t)+\lambda_{0} h_{2}(t)+\lambda_{0} h_{3}(t)
$$

where

$$
\begin{aligned}
h_{1}(t) & =\int_{0}^{\infty} \bar{F}_{Z}(x) \bar{F}_{Z}(x+t) d x, \\
h_{2}(t) & =\sum_{k=1}^{\infty} c(k) \int_{0}^{\infty} \bar{F}_{Z}(x) \mathbb{P}\left(Z>x+t+T_{k}\right) d x, \\
h_{3}(t) & =\sum_{k=1}^{\infty} c(k) \int_{0}^{\infty} \bar{F}_{Z}(x) \mathbb{P}\left(T_{k}<x+t \leq T_{k}+Z\right) d x .
\end{aligned}
$$

Note that $V(\cdot) \in \mathcal{R}_{2 H-2}$ by Lemma 2.3 and $2 H-2 \in(-1,0)$. Then Lemma 6.1 (c)-(e) give

$$
g_{2}(t) \sim \lambda_{0} h_{3}(t) \sim \lambda_{0}(\mathbb{E}(Z))^{2} V(t) \quad \text { as } t \rightarrow \infty
$$

Let $\gamma>3-2 H$. Then (6.12), (6.13) and (6.15) result in (a). If $\gamma<3-2 H$ then $\bar{F}_{Z} \in \mathcal{R}_{-\gamma}$ and

$$
g_{1}(t) \sim \frac{\lambda}{\gamma-1} t \bar{F}_{Z}(t) \quad \text { as } t \rightarrow \infty
$$

The reason is that

$$
\mathbb{E}\left(Z_{1} \wedge\left(Z_{2}-t\right)_{+}\right) \leq \mathbb{E}\left(Z_{1}\right) \mathbb{P}\left(Z_{2}>t\right)=o\left(t \bar{F}_{Z}(t)\right) \quad \text { as } t \rightarrow \infty
$$

and by Karamata's Theorem

$$
\mathbb{E}(Z-t)_{+} \sim \frac{1}{\gamma-1} t \bar{F}_{Z}(t) \quad \text { as } t \rightarrow \infty
$$


Thus, (b) follows from (6.12), (6.15) and (6.16).

\section{Proof of Proposition 3.2.}

We have by Lemma 2.1 (d) that

$$
\operatorname{Var}(A(t))=\int_{-t}^{t}\left[\min (t, t-x)-(-x)_{+}\right] g(x) d x=2 \int_{0}^{t}(t-x) g(x) d x .
$$

Let $\gamma>3-2 H$. Then from Lemma 6.2 we know that $g \in \mathcal{R}_{2 H-2}$. Hence, by Karamata's Theorem

$$
\operatorname{Var}(A(t)) \sim \frac{2}{2 H-1} t^{2} g(t)-\frac{1}{H} t^{2} g(t) \sim \frac{(\mathbb{E}(Z))^{2}}{(2 H-1) H} \lambda_{0} t^{2} V(t) \quad \text { as } t \rightarrow \infty
$$

follows. In the case $\gamma<3-2 H$ the same arguments lead to the result.

Proof of Theorem 3.4. First, we show (3.1). By Lemma 2.1 (b) we have with $f=\mathbf{1}_{(0,1]}$, $g=\mathbf{1}_{(h, h+1]}$ (cf. Faÿ et al. [14], equation (2.9)) that

$$
\begin{aligned}
\gamma_{N}(h) & =\lambda_{0} \int_{0}^{1} \sum_{k=1}^{\infty} c(k)\left[\mathbb{P}\left(T_{k} \leq v+h\right)-\mathbb{P}\left(T_{k} \leq v+h-1\right)\right] d v \\
& =\lambda_{0} \int_{0}^{1}[G(v+h)-G(v+h-1)] d v .
\end{aligned}
$$

Since $G(u)-G(u-1) \sim V(u) \in \mathcal{R}_{2 H-2}$ as $u \rightarrow \infty$ by Lemma 2.3 (d) we obtain by Embrechts et al. [13], Lemma 1.3.5 (a), that

$$
\gamma_{N}(h) \sim \lambda_{0} V(h) \quad \text { as } h \rightarrow \infty .
$$

Next, we investigate the asymptotic behavior of $\gamma_{A}$. Here, Lemma 2.1 (d) gives

$$
\gamma_{A}(h)=\int_{h-1}^{h+1}\left[\min (1, h+1-x)-(h-x)_{+}\right] g(x) d x=\int_{-1}^{1} g(z+h)(1-|z|) d z .
$$

Further, $g \in \mathcal{R}_{2 H-2}$ if $\gamma>3-2 H$ and $g \in \mathcal{R}_{1-\gamma}$ if $\gamma<3-2 H$, respectively (by Lemma 6.2) such that by Embrechts et al. [13], Lemma 1.3.5 (a), we obtain

$$
\gamma_{A}(h) \sim g(h) \int_{-1}^{1}(1-|z|) d z=g(h) \quad \text { as } h \rightarrow \infty,
$$

which proves the Theorem.

\subsection{Proofs of Section 4}

For the proof of Theorem 4.1 we use the following notation. Let

$$
b_{n}:=\sqrt{n M_{n} \bar{F}\left(M_{n}\right)^{-2} \mathbb{P}\left(K>\bar{F}_{X}\left(M_{n}\right)^{-1}\right)}=\frac{1}{\sqrt{D(\alpha, \beta)(\alpha-1)^{-1}}} \sqrt{n M_{n}^{2} V\left(M_{n}\right)}, \quad n \in \mathbb{N},(6.18)
$$

where $V$ and $D(\alpha, \beta)$ are given in (2.15) and (2.16), respectively, and

$$
\begin{aligned}
\sigma^{2} & :=\frac{2 \lambda_{0}}{2+\beta-\alpha} \int_{0}^{\infty} y^{-(2+\beta-\alpha) / \beta} \mathbb{P}\left(S_{1 / \beta}(1) \leq y\right) d y+ \\
& +\lambda_{0} \int_{0}^{\infty} \mathbb{E}\left(\frac{2}{2-\alpha} I(w+1)^{2-\alpha}+\frac{2}{\alpha-1} I(w) I(w+1)^{1-\alpha}-\frac{2}{(2-\alpha)(\alpha-1)} I(w)^{2-\alpha}\right) d w
\end{aligned}
$$


where $\left(S_{1 / \beta}(t)\right)_{t \geq 0}$ is a $1 / \beta$-stable Lévy process which satisfies

$$
\left(\frac{\sum_{k=1}^{\lfloor n t\rfloor} X_{k}}{\bar{F}_{X}^{\leftarrow}\left(n^{-1}\right)}\right)_{t \geq 0} \Longrightarrow\left(S_{1 / \beta}(t)\right)_{t \geq 0} \quad \text { as } n \rightarrow \infty \text { in }\left(\mathbb{D}[0, \infty), J_{1}\right),
$$

and

$$
I(w)=\inf \left\{t \geq 0: S_{1 / \beta}(t)>w\right\} \quad \text { for } w>0 .
$$

Furthermore, for the proof of Theorem 4.1 we require the next Lemma.

Lemma 6.3 Let the assumptions and the notation of Theorem 4.1 hold. Further, let $\left(b_{n}\right)$ be given as in (6.18) and $\sigma^{2}$ be given as in (6.19), respectively. Then

$$
n \operatorname{Var}\left(N\left(0, M_{n}\right]\right) \sim \sigma^{2} b_{n}^{2} \quad \text { as } n \rightarrow \infty,
$$

and in particular,

$$
\sigma^{2}=\frac{\lambda_{0}}{(2 H-1) H} D(\alpha, \beta)(\alpha-1)^{-1}=\frac{\Gamma\left(1-\beta^{-1}\right)^{2-\alpha} \Gamma(3-\alpha)}{\beta(\alpha-1)(2 H-1) H \Gamma(2 H)} .
$$

Proof. We decompose $N\left(0, M_{n} t\right]$ in the independent components

$$
N\left(0, M_{n} t\right]=N^{+}\left(0, M_{n} t\right]+N^{-}\left(0, M_{n} t\right]
$$

where $N^{+}\left(0, M_{n} t\right]$ is the number of arrivals of packets in $\left(0, M_{n} t\right]$ belonging to a cluster initiated in $\left(0, M_{n} t\right]$ and $N^{-}\left(0, M_{n} t\right]$ is the number of arrivals of packets in $\left(0, M_{n} t\right]$ belonging to a cluster initiated in $(-\infty, 0]$, respectively, i.e.

$$
\begin{aligned}
& N^{+}\left(0, M_{n} t\right]=\#\left\{(m, k) \in \mathbb{Z}^{2}: \Gamma_{m} \in\left(0, M_{n} t\right], \Gamma_{m, k} \in\left(0, M_{n} t\right], k \in\left\{0, \ldots, K_{m}\right\}\right\}, \\
& N^{-}\left(0, M_{n} t\right]=\#\left\{(m, k) \in \mathbb{Z}^{2}: \Gamma_{m} \in(-\infty, 0], \Gamma_{m, k} \in\left(0, M_{n} t\right], k \in\left\{0, \ldots, K_{m}\right\}\right\} .
\end{aligned}
$$

Furthermore,

$$
\frac{N^{ \pm}\left(0, M_{n} t\right]-\mathbb{E}\left(N^{ \pm}\left(0, M_{n} t\right]\right)}{b_{n}}
$$

are infinitely divisible with characteristic triplet $\left(0,0, \widetilde{\nu}_{n, t}^{ \pm}\right)$where for $A \in \mathcal{B}(\mathbb{R})$ :

$$
\begin{aligned}
& \widetilde{\nu}_{n, t}^{+}(A)=\lambda_{0} \int_{0}^{M_{n} t} \mathbb{P}\left(b_{n}^{-1} \sum_{k=0}^{K} \varepsilon_{T_{k}}[0, u] \in A\right) d u, \\
& \widetilde{\nu}_{n, t}^{-}(A)=\lambda_{0} \int_{0}^{\infty} \mathbb{P}\left(b_{n}^{-1} \sum_{k=0}^{K} \varepsilon_{T_{k}}\left(u, u+M_{n} t\right] \in A\right) d u ;
\end{aligned}
$$

for more details see Fasen and Samorodnitsky [15], p. 405. Thus, Sato [31], Example 25.12, and the independence of $N^{+}$and $N^{-}$result in

$$
\begin{aligned}
\operatorname{Var}\left(\frac{N\left(0, M_{n} t\right]}{b_{n}}\right) & =\operatorname{Var}\left(\frac{N^{+}\left(0, M_{n} t\right]}{b_{n}}\right)+\operatorname{Var}\left(\frac{N^{-}\left(0, M_{n} t\right]}{b_{n}}\right) \\
& =\int_{0}^{\infty} x^{2} \widetilde{\nu}_{n, t}^{+}(d x)+\int_{0}^{\infty} x^{2} \widetilde{\nu}_{n, t}^{-}(d x) .
\end{aligned}
$$


Define $\nu_{n, t}^{ \pm}:=n \widetilde{\nu}_{n, t}^{ \pm}$. Now, Lemma 5.1 and Lemma 5.2 of Fasen and Samorodnitsky [15] (which are also valid for $\epsilon=\infty$ ) give

$$
\frac{n}{b_{n}^{2}} \operatorname{Var}\left(N\left(0, M_{n} t\right]\right)=\int_{0}^{\infty} x^{2} \nu_{n, t}^{+}(d x)+\int_{0}^{\infty} x^{2} \nu_{n, t}^{-}(d x) \sim t^{2 H} \sigma_{+}^{2}+t^{2 H} \sigma_{-}^{2} \quad \text { as } n \rightarrow \infty,
$$

where $\sigma_{+}^{2}+\sigma_{-}^{2}=\sigma^{2}$.

Proof of Theorem 4.1. We start with the proof of (b).

(b) Fasen and Samorodnitsky [15], Proposition 5.1, says that

$$
\frac{1}{b_{n}} \sum_{i=1}^{n}\left[N_{i}\left(0, M_{n} t\right]-\lambda M_{n} t\right] \stackrel{\mathrm{FDD}}{\Longrightarrow}\left(\sigma B_{H}(t)\right)_{t \geq 0} \quad \text { as } n \rightarrow \infty,
$$

which gives together with Lemma 6.3 the convergence of the finite dimensional distributions in (b). For the proof of the tightness we use Billingsley [3], Theorem 12.3. Therefore, it is sufficient to prove

$$
\mathbb{E}\left|N_{n}(t)\right|^{2} \leq C t^{2 H-\delta} \quad \text { for } t \in[0,1]
$$

some $C>0,0<\delta<2 H-1$, where

$$
N_{n}(t):=\left(n \operatorname{Var}\left(N\left(0, M_{n}\right]\right)\right)^{-\frac{1}{2}} \sum_{i=1}^{n}\left[N_{i}\left(0, M_{n} t\right]-\lambda M_{n} t\right] \quad \text { for } t \in[0,1],
$$

is stationary. Note that

$$
\mathbb{E}\left|N_{n}(t)\right|^{2}=\frac{\operatorname{Var}\left(N\left(0, M_{n} t\right]\right)}{\operatorname{Var}\left(N\left(0, M_{n}\right]\right)} .
$$

Proposition 3.1 gives $\operatorname{Var}(N(0, \cdot]) \in \mathcal{R}_{2 H}$. Thus, Potter's Theorem (Bingham et al. [6], Theorem 1.5.6) and $t \in[0,1]$ result in (6.21).

(a) Let $\nu_{n, t}$ be the Lévy measure of $N_{n}(t)$, and $\widehat{\nu}_{n, t}$ be the Lévy measure of

$$
\frac{N\left(0, M_{n} t\right]-\lambda M_{n} t}{\sqrt{\operatorname{Var}\left(N\left(0, M_{n}\right]\right)}}
$$

Then

$$
\widehat{\nu}_{n, t}(\cdot)=\frac{1}{n} \nu_{n, t}\left(\frac{1}{\sqrt{n}} \cdot\right)
$$

We have to prove

(i) $\widehat{\nu}_{n, t}(\cdot)=\frac{1}{n} \nu_{n, t}\left(\frac{1}{\sqrt{n}} \cdot\right) \stackrel{\nu}{\Longrightarrow} 0$ on $(0, \infty]$ as $n \rightarrow \infty$

(ii) $\lim _{n \rightarrow \infty} \int_{\{|x| \leq 1\}} x^{2} \widehat{\nu}_{n, t}(d x)=\lim _{n \rightarrow \infty} \int_{\left\{|z| \leq n^{-1 / 2}\right\}} z^{2} \nu_{n, t}(d z)=t^{2 H}$,

(iii) $\lim _{n \rightarrow \infty} \int_{\{|x|>1\}} x \widehat{\nu}_{n, t}(d x)=\lim _{n \rightarrow \infty} \frac{1}{\sqrt{n}} \int_{\left\{|z|>n^{-1 / 2}\right\}} z \nu_{n, t}(d z)=0$,

which reflects the convergence of the characteristic triplet of the infinitely divisible random variable $N_{n}(t)$ to the characteristic triplet of a Gaussian random variable with mean 0 and variance $t^{2 H}$. Then the claim follows by Kallenberg [20], Theorem 15.14.

The proof of (i)-(iii) can be done step by step as in Fasen and Samorodnitsky [15], Lemma 5.1 and Lemma 5.2 using $\lim _{n \rightarrow \infty} n^{-\frac{1}{2}} \bar{F}\left(M_{n}\right) b_{n}=\infty$ and Lemma 6.3. 


\subsection{Proofs of Section 5}

For the proof of Theorem 5.1 we require the following Lemma.

Lemma 6.4 Let Assumption $A, B$ and $C$ hold, and define

$$
\begin{aligned}
& A^{(1)}(t)=\sum_{l \in \mathbb{Z}} \mathbf{1}_{\left\{\mathcal{I}_{l} \leq 0\right\}}\left(\left(\mathcal{T}_{l}+\mathcal{Z}_{l}\right)_{+} \wedge t\right), \quad t \geq 0, \\
& A^{(2)}(t)=\sum_{l \in \mathbb{Z}} \mathbf{1}_{\left\{0<\mathcal{T}_{l} \leq t\right\}}\left(\mathcal{T}_{l}+\mathcal{Z}_{l}-t\right)_{+}, \quad t \geq 0 .
\end{aligned}
$$

(a) Suppose $\mathbb{E}\left(Z^{2}\right)<\infty$. Then

$$
0 \leq \mathbb{E}\left(A^{(1)}(t)\right)=\mathbb{E}\left(A^{(2)}(t)\right) \leq \frac{\lambda}{2} \mathbb{E}\left(Z^{2}\right), \quad t \geq 0 .
$$

(b) Suppose $1<\gamma<2$. Then

$$
\mathbb{E}\left(A^{(1)}(t)\right)=\mathbb{E}\left(A^{(2)}(t)\right) \sim \lambda(2-\gamma)^{-1}(\gamma-1)^{-1} t^{2} \bar{F}_{Z}(t) \quad \text { as } t \rightarrow \infty .
$$

Proof. Let $f(s, z)=\mathbf{1}_{\{s \leq 0\}}\left((s+z)_{+} \wedge t\right)$. Then we obtain by Lemma 2.1 (a)

$$
\begin{aligned}
\mathbb{E}\left(A^{(1)}(t)\right) & =\lambda \int_{0}^{\infty} \int_{-\infty}^{0}\left((s+z)_{+} \wedge t\right) d s F_{Z}(d z) \\
& =\lambda \int_{0}^{\infty} \int_{-\infty}^{0} \int_{0}^{t} \mathbf{1}_{\{x<z+s\}} d x d s F_{Z}(d z) \\
& =\lambda \int_{0}^{t} \int_{x}^{\infty} \bar{F}_{Z}(s) d s d x .
\end{aligned}
$$

(a) If $\mathbb{E}\left(Z^{2}\right)<\infty$ then

$$
\mathbb{E}\left(A^{(1)}(t)\right)=\lambda \int_{0}^{\infty} \bar{F}_{Z}(s) \int_{0}^{s \wedge t} d x d s \leq \lambda \int_{0}^{\infty} \bar{F}_{Z}(s) s d s=\frac{\lambda}{2} \int_{0}^{\infty} \bar{F}_{Z}(\sqrt{z}) d z=\frac{\lambda}{2} \mathbb{E}\left(Z^{2}\right) .
$$

(b) Define $h(x)=\int_{x}^{\infty} \bar{F}_{Z}(s) d s$. Then by Karamata's Theorem we know that $h \in \mathcal{R}_{1-\gamma}$ and $h(x) \sim(\gamma-1)^{-1} x \bar{F}_{Z}(x)$ as $x \rightarrow \infty$. Thus, again with Karamata's Theorem we have

$$
\mathbb{E}\left(A^{(1)}(t)\right)=\lambda \int_{0}^{t} h(x) d x \sim \lambda(2-\gamma)^{-1} t h(t) \sim \lambda(2-\gamma)^{-1}(\gamma-1)^{-1} t^{2} \bar{F}_{Z}(t) \quad \text { as } t \rightarrow \infty .
$$

The equality $\mathbb{E}\left(A^{(1)}(t)\right)=\mathbb{E}\left(A^{(2)}(t)\right)$ was proven in Mikosch and Samorodnitsky [26], p. 905.

Proof of Theorem 5.1. We use the decomposition

$$
\begin{aligned}
A\left(M_{n} t\right) & =\sum_{l=1}^{N\left(0, M_{n} t\right]} \mathcal{Z}_{l}+\sum_{l \in \mathbb{Z}} \mathbf{1}_{\left\{\mathcal{T}_{l} \leq 0\right\}}\left(\left(\mathcal{T}_{l}+\mathcal{Z}_{l}\right)_{+} \wedge M_{n} t\right)-\sum_{l \in \mathbb{Z}} \mathbf{1}_{\left\{0<\mathcal{T}_{l} \leq M_{n} t\right\}}\left(\mathcal{T}_{l}+\mathcal{Z}_{l}-M_{n} t\right)_{+} \\
& =: A^{(0)}\left(M_{n} t\right)+A^{(1)}\left(M_{n} t\right)-A^{(2)}\left(M_{n} t\right),
\end{aligned}
$$

where $A^{(0)}\left(M_{n} t\right)$ represents the amount of data whose transmission is initiated in $\left(0, M_{n} t\right]$. On the other hand, $A^{(1)}\left(M_{n} t\right)$ reflects the data in $\left(0, M_{n} t\right]$ whose transmission was initiated before 0 . 
Finally, $A^{(2)}\left(M_{n} t\right)$ is the data transfer going on after $M_{n} t$ but which was started in $\left(0, M_{n} t\right]$. Then

$$
\begin{aligned}
A\left(M_{n} t\right)-\lambda M_{n} t \mathbb{E}(Z) & =\left[\sum_{l=1}^{N\left(0, M_{n} t\right]} \mathcal{Z}_{l}-\lambda M_{n} t \mathbb{E}(Z)\right]+\left[A^{(1)}\left(M_{n} t\right)-A^{(2)}\left(M_{n} t\right)\right] \\
& =: S_{1}\left(M_{n} t\right)+S_{2}\left(M_{n} t\right) .
\end{aligned}
$$

On the one hand, applying Theorem 4.1 we know that

$$
\left(\frac{N\left(0, M_{n} t\right]-\lambda M_{n} t}{\sqrt{\operatorname{Var}\left(N\left(0, M_{n}\right]\right)}}\right)_{t \geq 0} \Longrightarrow\left(B_{H}(t)\right)_{t \geq 0} \quad \text { as } n \rightarrow \infty \text { in } \mathbb{C}[0,1] .
$$

On the other hand, by (2.11)

$$
\left(\frac{\sum_{l=1}^{\left\lfloor M_{n} t\right\rfloor}\left[\mathcal{Z}_{l}-\mathbb{E}(Z)\right]}{a\left(M_{n}\right)}\right)_{t \geq 0} \Longrightarrow\left(S_{\gamma}(t)\right)_{t \geq 0} \quad \text { as } n \rightarrow \infty \text { in }\left(\mathbb{D}[0,1], J_{1}\right) .
$$

(a) Furthermore, by Proposition 3.1 and Potter's Theorem there exists for some $0<\epsilon<H-\gamma^{-1}$ a $C_{1}>0$ such that for $M_{n}$ large

$$
\frac{a^{2}\left(M_{n}\right)}{\operatorname{Var}\left(N\left(0, M_{n}\right]\right)} \leq C_{1} \frac{M_{n}^{\frac{2}{\gamma}+\epsilon}}{M_{n}^{2 H-\epsilon}}=C_{1} M_{n}^{\frac{2}{\gamma}-2 H+2 \epsilon}
$$

which tends to 0 as $n \rightarrow \infty$. Hence,

$$
\left(\frac{\sum_{l=1}^{\left\lfloor M_{n} t\right\rfloor}\left[\mathcal{Z}_{l}-\mathbb{E}(Z)\right]}{\sqrt{\operatorname{Var}\left(N\left(0, M_{n}\right]\right)}}\right)_{t \geq 0} \stackrel{\mathbb{P}}{\longrightarrow}(0)_{t \geq 0} \quad \text { as } n \rightarrow \infty \text { in }\left(\mathbb{D}[0,1], J_{1}\right) .
$$

Then (6.23), (6.25) and Whitt [34], Corollary 13.3.2 result in

$$
\left(\frac{S_{1}\left(M_{n} t\right)}{\sqrt{\operatorname{Var}\left(N\left(0, M_{n}\right]\right)}}\right)_{t \geq 0} \Longrightarrow\left(\mathbb{E}(Z) B_{H}(t)\right)_{t \geq 0} \quad \text { as } n \rightarrow \infty \text { in }\left(\mathbb{D}[0,1], J_{1}\right) \text {. }
$$

Note that $2-\gamma-H<0$ since $\gamma>H^{-1}>1$. Moreover, by Proposition 3.1, Lemma 6.4 and Potter's Theorem there exists for some $0<\epsilon<\frac{H+\gamma-2}{2}$ a constant $C_{2}>0$ such that for $t>0$ and $M_{n}$ large

$$
\frac{\mathbb{E}\left(A^{(j)}\left(M_{n} t\right)\right)}{\sqrt{\operatorname{Var}\left(N\left(0, M_{n}\right]\right)}} \leq C_{2} \frac{\left(M_{n} t\right)^{2-\gamma+\epsilon}}{M_{n}^{H-\epsilon}} \leq C_{2} M_{n}^{2-\gamma-H+2 \epsilon}, \quad j=1,2,
$$

which tends to 0 as $n$ tends to $\infty$. Hence, Markov's inequality gives for any $\delta>0$,

$$
\lim _{n \rightarrow \infty} \mathbb{P}\left(\frac{A^{(j)}\left(M_{n} t\right)}{\sqrt{\operatorname{Var}\left(N\left(0, M_{n}\right]\right)}}>\delta\right)=0,
$$

which means

$$
\frac{S_{2}\left(M_{n} t\right)}{\sqrt{\operatorname{Var}\left(N\left(0, M_{n}\right]\right)}} \stackrel{\mathbb{P}}{\longrightarrow} 0 \quad \text { as } n \rightarrow \infty
$$


The conclusion follows then from (6.22), (6.26) and (6.27).

In the case $\gamma>3-2 H$ we can prove the convergence in $\mathbb{C}[0,1]$. Thus, we show that $\left(A_{n}(t)\right)_{t \in[0,1]}$, where

$$
A_{n}(t)=\frac{A\left(M_{n} t\right)-\lambda M_{n} t \mathbb{E}(Z)}{\sqrt{\operatorname{Var}\left(A\left(M_{n}\right)\right)}},
$$

is tight by Billingsley [3], Theorem 12.3. As in Theorem 4.1 it is sufficient to prove that there exists a $0<\delta<2 H-1$ such that

$$
\mathbb{E}\left|A_{n}(t)\right|^{2} \leq C_{3} t^{2 H-\delta} \quad \text { for } t \in[0,1]
$$

and some $C_{3}>0$. We have

$$
\mathbb{E}\left|A_{n}(t)\right|^{2}=\frac{\operatorname{Var}\left(A\left(M_{n} t\right)\right)}{\operatorname{Var}\left(A\left(M_{n}\right)\right)} .
$$

Since Proposition 3.2 says that $\operatorname{Var}(A(\cdot)) \in \mathcal{R}_{2 H}$, Potter's Theorem shows (6.28) as well.

(b) Here we have again by Potter's Theorem that for some constant $C_{4}>0$ :

$$
\frac{\operatorname{Var}\left(N\left(0, M_{n}\right]\right)}{a^{2}\left(M_{n}\right)} \leq C_{4} \frac{M_{n}^{2 H+\epsilon}}{M_{n}^{\frac{2}{\gamma}-\epsilon}}=C_{4} M_{n}^{2 H-\frac{2}{\gamma}+2 \epsilon},
$$

which tends to 0 as $n \rightarrow \infty$. Hence,

$$
\left(\frac{N\left(0, M_{n} t\right]-\lambda M_{n} t}{a\left(M_{n}\right)}\right)_{t \geq 0} \Longrightarrow(0)_{t \geq 0} \quad \text { as } n \rightarrow \infty \text { in } \mathbb{C}[0,1] .
$$

Again Whitt [34], Corollary 13.3.2, (6.24) and (6.29) give

$$
\left(\frac{S_{1}\left(M_{n} t\right)}{a\left(M_{n}\right)}\right)_{t \geq 0} \Longrightarrow\left(\lambda^{\frac{1}{\gamma}} S_{\gamma}(t)\right)_{t \geq 0} \quad \text { as } n \rightarrow \infty \text { in }\left(\mathbb{D}[0,1], J_{1}\right)
$$

Finally, by Lemma 6.4 and Potter's Theorem there exists for some $0<\epsilon<\frac{(\gamma-1)^{2}}{2 \gamma}$ a constant $C_{5}>0$ such that

$$
\frac{\mathbb{E}\left(A^{(j)}\left(M_{n} t\right)\right)}{a\left(M_{n}\right)} \leq C_{5} \frac{M_{n}^{2-\gamma+\epsilon}}{M_{n}^{\frac{1}{\gamma}-\epsilon}}=C_{5} M_{n}^{-\frac{(\gamma-1)^{2}}{\gamma}+2 \epsilon} \longrightarrow 0 \quad \text { as } n \rightarrow \infty, \quad j=1,2 .
$$

Thus, for any $t \in[0,1]$,

$$
\frac{S_{2}\left(M_{n} t\right)}{a\left(M_{n}\right)} \stackrel{\mathbb{P}}{\longrightarrow} 0 \quad \text { as } n \rightarrow \infty,
$$

and we obtain the result by (6.22), (6.30) and (6.31).

Remark 6.5 In both (a) and (b) we saw that the properly normalized process $\left(S_{1}\left(M_{n} t\right)\right)_{t \geq 0}$ converges in $\left(\mathbb{D}[0,1], J_{1}\right)$. However, the proof of the tightness of the normalized process $\left(S_{2}\left(M_{n} t\right)\right)_{t \geq 0}$ can be difficult independently of the topology in $\mathbb{D}[0,1]$. Although the tightness of the scaled process $\left(A^{(1)}\left(M_{n} t\right)\right)$ is by $A^{(1)}\left(M_{n} t\right) \leq A^{(1)}\left(M_{n}\right), 0 \leq t \leq 1$, obvious, the tightness of $\left(A^{(2)}\left(M_{n} t\right)\right)$ is involved. 


\section{Proof of Theorem 5.3.}

(a) We start to prove the convergence of the finite dimensional distributions. Therefore, we use for $i=1, \ldots, n$ the decomposition

$$
\begin{aligned}
A_{i}(t) & =\sum_{l=1}^{N_{i}(0, t]} \mathcal{Z}_{l}^{(i)}+\sum_{l \in \mathbb{Z}} \mathbf{1}_{\left\{\mathcal{T}_{l}^{(i)} \leq 0\right\}}\left(\left(\mathcal{T}_{l}^{(i)}+\mathcal{Z}_{l}^{(i)}\right)_{+} \wedge t\right)-\sum_{l \in \mathbb{Z}} \mathbf{1}_{\left\{0<\mathcal{T}_{l}^{(i)} \leq t\right\}}\left(\mathcal{T}_{l}^{(i)}+\mathcal{Z}_{l}^{(i)}-t\right)_{+} \\
& =: \quad A_{i}^{(0)}(t)+A_{i}^{(1)}(t)-A_{i}^{(2)}(t) .
\end{aligned}
$$

Then

$$
\begin{aligned}
S_{n, M_{n}}(t) & =\sum_{i=1}^{n}\left[A_{i}\left(M_{n} t\right)-\lambda M_{n} t \mathbb{E}(Z)\right] \\
& =\sum_{i=1}^{n}\left[\sum_{l=1}^{N_{i}\left(0, M_{n} t\right]} \mathcal{Z}_{l}^{(i)}-\lambda M_{n} t \mathbb{E}(Z)\right]+\sum_{i=1}^{n}\left[A_{i}^{(1)}\left(M_{n} t\right)-A_{i}^{(2)}\left(M_{n} t\right)\right] \\
& =: S_{n, M_{n}}^{(1)}(t)+S_{n, M_{n}}^{(2)}(t) .
\end{aligned}
$$

First, we investigate the asymptotic behavior of $\left(S_{n, M_{n}}^{(1)}(t)\right)_{t \geq 0}$. A conclusion of Theorem 4.1 and Proposition 3.2 is that

$$
\left(\frac{\sum_{i=1}^{n}\left[N_{i}\left(0, M_{n} t\right]-\lambda M_{n} t\right]}{\sqrt{n \operatorname{Var}\left(A\left(M_{n}\right)\right)}}\right)_{t \geq 0} \Longrightarrow\left(\mathbb{E}(Z)^{-1} B_{H}(t)\right)_{t \geq 0} \quad \text { as } n \rightarrow \infty \text { in } \mathbb{C}[0,1] .
$$

Next, note that

$$
\left(\sum_{i=1}^{n}\left[\sum_{l=1}^{N_{i}\left(0, M_{n} t\right]} \mathcal{Z}_{l}^{(i)}-\lambda M_{n} t \mathbb{E}(Z)\right]\right)_{t \geq 0} \stackrel{d}{=}\left(\sum_{l=1}^{\sum_{i=1}^{n} N_{i}\left(0, M_{n} t\right]} \mathcal{Z}_{l}-\lambda n M_{n} t \mathbb{E}(Z)\right)_{t \geq 0}
$$

and that by $(2.11)$

$$
\left(\frac{\sum_{l=1}^{\left\lfloor n M_{n} t\right\rfloor}\left[\mathcal{Z}_{l}-\mathbb{E}(Z)\right]}{a\left(n M_{n}\right)}\right)_{t \geq 0} \Longrightarrow\left(S_{\gamma}(t)\right)_{t \geq 0} \quad \text { as } n \rightarrow \infty \text { in }\left(\mathbb{D}[0,1], J_{1}\right) .
$$

By assumption (a) we have that there exists a constant $C_{1} \in(0, \infty)$ such that

$$
n^{\frac{1}{\gamma-1}-\epsilon} M_{n}^{-1} \leq C_{1} \quad \text { for } n \in \mathbb{N} .
$$

Moreover, by Proposition 3.2 and Potter's Theorem we have that for any $\epsilon>0$ there exist $C_{2}>0$ and $n_{0} \in \mathbb{N}$ such that for $n \geq n_{0}$ :

$$
\frac{a\left(n M_{n}\right)^{2}}{n \operatorname{Var}\left(A\left(M_{n}\right)\right)} \leq C_{2} \frac{\left(n M_{n}\right)^{\frac{2}{\gamma}+\epsilon}}{n M_{n}^{2 H-\epsilon}}=C_{2}\left(n^{\frac{1}{\gamma-1}-\epsilon} M_{n}^{-1}\right)^{\frac{2 H \gamma-2}{\gamma}-2 \epsilon} n^{\frac{3-2 H-\gamma}{\gamma-1}+\epsilon^{\prime}},
$$

where $\epsilon^{\prime}=\frac{2 \epsilon}{\gamma-1}+\epsilon \frac{2 H \gamma-2}{\gamma}-2 \epsilon^{2}+\epsilon$. For small $\epsilon$ the right hand side of (6.37) tends to 0 by (6.36). Hence, Corollary 13.3.2 of Whitt [34], (6.33), (6.35) and (6.37) result in

$$
\left(\frac{\sum_{l=1}^{\sum_{i=1}^{n} N_{i}\left(0, M_{n} t\right]} \mathcal{Z}_{l}-\lambda n M_{n} t \mathbb{E}(Z)}{\sqrt{n \operatorname{Var}\left(A\left(M_{n}\right)\right)}}\right)_{t \geq 0} \Longrightarrow\left(B_{H}(t)\right)_{t \geq 0} \text { as } n \rightarrow \infty \text { in }\left(\mathbb{D}[0,1], J_{1}\right)
$$


and finally by (6.34) in

$$
\left(\frac{S_{n, M_{n}}^{(1)}(t)}{\sqrt{n \operatorname{Var}\left(A\left(M_{n}\right)\right)}}\right)_{t \geq 0} \Longrightarrow\left(B_{H}(t)\right)_{t \geq 0} \text { as } n \rightarrow \infty \text { in }\left(\mathbb{D}[0,1], J_{1}\right) \text {. }
$$

Since $\gamma>3-2 H$ is equivalent to

$$
-\frac{1}{2}(\gamma-1)>2-H-\gamma
$$

there exists an $\epsilon>0$ such that

$$
-\frac{1}{2}(\gamma-1) \frac{1}{1-\epsilon(\gamma-1)}-2 \epsilon>2-H-\gamma .
$$

Finally, by Potter's Theorem, Proposition 3.2, Lemma 6.4 and (6.36) we have for some $C_{3}, C_{4}>0$ and $n$ large

$$
\begin{aligned}
\frac{n \mathbb{E}\left(A^{(j)}\left(M_{n} t\right)\right)}{\sqrt{n \operatorname{Var}\left(A\left(M_{n}\right)\right)}} & \leq C_{3} \frac{n\left(M_{n} t\right)^{2-\gamma+\epsilon}}{\left(n M_{n}^{2 H-2 \epsilon}\right)^{\frac{1}{2}}} \\
& \leq C_{3}\left(n^{\frac{1}{\gamma-1}-\epsilon} M_{n}^{-1}\right)^{\frac{\gamma-1}{2(1-\epsilon(\gamma-1))}} M_{n}^{-\epsilon} t^{2-\gamma+\epsilon} \\
& \leq C_{4} M_{n}^{-\epsilon}, \quad j=1,2,
\end{aligned}
$$

which tends to 0 as $n \rightarrow \infty$. Then Markov's inequality and (6.39) result in

$$
\lim _{n \rightarrow \infty} \mathbb{P}\left(\frac{\sum_{i=1}^{n} A_{i}^{(j)}\left(M_{n} t\right)}{\sqrt{n \operatorname{Var}\left(A\left(M_{n}\right)\right)}}>\delta\right)=0 \quad \text { for any } \delta>0,
$$

and consequently, as $n \rightarrow \infty$,

$$
\frac{1}{\sqrt{n \operatorname{Var}\left(A\left(M_{n}\right)\right)}} S_{n, M_{n}}^{(2)}(t)=\frac{1}{\sqrt{n \operatorname{Var}\left(A\left(M_{n}\right)\right)}} \sum_{i=1}^{n}\left[A_{i}^{(1)}\left(M_{n} t\right)-A_{i}^{(2)}\left(M_{n} t\right)\right] \stackrel{\mathbb{P}}{\longrightarrow} 0 .
$$

Thus, (6.32), (6.38), (6.40) and Theorem 25.4 of Billingsley [4] give

$$
\left(\frac{1}{\sqrt{n \operatorname{Var}\left(A\left(M_{n}\right)\right)}} \sum_{i=1}^{n}\left[A_{i}\left(M_{n} t\right)-\lambda M_{n} t \mathbb{E}(Z)\right]\right)_{t \geq 0} \stackrel{\text { FDD }}{\longrightarrow}\left(B_{H}(t)\right)_{t \geq 0} \quad \text { as } n \rightarrow \infty .
$$

The tightness follows as in Theorem 5.1 by Proposition 3.2 and Billingsley [3], Theorem 12.3.

(b) In the fast growth case the result follows from $3-2 H>\gamma$, Lemma 6.2 (a), and Mikosch and Samorodnitsky [26], Theorem 4.2 , where we have $\mathbb{E}|M(0)|^{2+\widetilde{\epsilon}}<\infty$ for some $\widetilde{\epsilon}>0$ by Lemma 4.1 of Mikosch and Samorodnitsky [26] and Faÿ et al. [14], p. 124.

Remark 6.6 If $\bar{F}_{Z} \in \mathcal{R}_{-\gamma}$ with $1<\gamma<2$ then similar computations as in Lemma 6.7 show that for some constant $C>0$ :

$$
\operatorname{Var}\left(A^{(1)}(t)\right) \sim C t^{3} \bar{F}_{Z}(t) \quad \text { as } t \rightarrow \infty .
$$

However, the second moment of $A^{(2)}(t)$ does not exist $\left(V_{2}(t)=\infty\right.$ in (6.6)) such that with our approach we can not neglect the continuity condition in Theorem 5.3 in general. 
Proof of Theorem 5.4. The proof follows as in Theorem 5.3 (a). The only difference is that we use instead of (6.37),

$$
\frac{a\left(n M_{n}\right)^{2}}{n \operatorname{Var}\left(A\left(M_{n}\right)\right)} \leq C \frac{n M_{n}}{n M_{n}^{2 H-\epsilon}}=C M_{n}^{1-2 H+\epsilon} \longrightarrow 0 \quad \text { as } n \rightarrow \infty,
$$

which does not depend on the growth rate, and instead of (6.39) that

$$
\frac{n \mathbb{E}\left(A^{(j)}\left(M_{n} t\right)-\mathbb{E}\left(A^{(j)}\left(M_{n} t\right)\right)\right)^{2}}{n \operatorname{Var}\left(A\left(M_{n}\right)\right)}=\frac{\operatorname{Var}\left(A^{(j)}\left(M_{n} t\right)\right)}{\operatorname{Var}\left(A\left(M_{n}\right)\right)} \leq C t \frac{M_{n}}{M_{n}^{2 H-\epsilon}} \stackrel{n \rightarrow \infty}{\longrightarrow} 0
$$

by an application of Lemma 6.7 below and $\mathbb{E}\left(A^{(1)}\left(M_{n} t\right)\right)=\mathbb{E}\left(A^{(2)}\left(M_{n} t\right)\right)$.

Lemma 6.7 Let Assumption $A, B$ and $C$ hold and $\mathbb{E}\left(Z^{2}\right)<\infty$.

(a) Let $A^{(1)}(t)=\sum_{l \in \mathbb{Z}} \mathbf{1}_{\left\{\mathcal{T}_{l} \leq 0\right\}}\left(\left(\mathcal{T}_{l}+\mathcal{Z}_{l}\right)_{+} \wedge t\right), t \geq 0$. Then there exist $C, t_{0}>0$ such that

$$
\operatorname{Var}\left(A^{(1)}(t)\right) \leq C t \quad \text { for } t \geq t_{0} .
$$

(b) Let $A^{(2)}(t)=\sum_{l \in \mathbb{Z}} \mathbf{1}_{\left\{0<\mathcal{T}_{l} \leq t\right\}}\left(\mathcal{T}_{l}+\mathcal{Z}_{l}-t\right)_{+}, t \geq 0$. Then there exist $C, t_{0}>0$ such that

$$
\operatorname{Var}\left(A^{(2)}(t)\right) \leq C t \quad \text { for } t \geq t_{0} .
$$

\section{Proof.}

(a) We use the decomposition (6.6) of the variance in

$$
\operatorname{Var}\left(A^{(1)}(t)\right)=V_{1}(t)+V_{2}(t)
$$

where $f(s, z)=g(s, z)=\mathbf{1}_{\{s \leq 0\}}\left((s+z)_{+} \wedge t\right)$. Without loss of generality we can assume $\lambda_{0}=1$. Then, with Fubini's Theorem

$V_{1}(t)$

$$
\begin{aligned}
& =\sum_{\substack{k=-\infty \\
k \neq 0}}^{\infty} c(|k|) \int_{\mathbb{R}^{\prime} \times \mathbb{R}_{+}^{2}} \mathbb{E}\left(\mathbf{1}_{\left\{s \leq 0, s+T_{k} \leq 0\right\}}\left(\left(s+z_{1}\right)_{+} \wedge t\right)\left(\left(s+T_{k}+z_{2}\right)_{+} \wedge t\right)\right) F_{Z}\left(d z_{1}\right) F_{Z}\left(d z_{2}\right) d s \\
& =2 \sum_{k=-\infty}^{-1} c(-k) \int_{\mathbb{R}_{-} \times \mathbb{R}_{+}^{2}} \int_{[0, t] \times[0, t]} \mathbb{E}\left(\mathbf{1}_{\left\{x<s+z_{1}\right\}} \mathbf{1}_{\left\{y<s+T_{k}+z_{2}\right\}}\right) d x d y F_{Z}\left(d z_{1}\right) F_{Z}\left(d z_{2}\right) d s \\
& =2 \sum_{k=1}^{\infty} c(k) \int_{\mathbb{R}_{+}} \int_{[0, t] \times[0, t]} \bar{F}_{Z}(x+v) \mathbb{P}\left(y<-v-T_{k}+Z\right) d x d y d v .
\end{aligned}
$$

By the monotonicity of $\bar{F}$ we have

$$
V_{1}(t) \leq 2 t \int_{0}^{t}\left[\int_{0}^{\infty} \bar{F}_{Z}(v)\left[\sum_{k=1}^{\infty} c(k) \mathbb{P}\left(Z>y+v+T_{k}\right)\right] d v\right] d y .
$$


A conclusion of Lemma 6.1 (d) with $\gamma_{1}=2$ is

$$
\begin{aligned}
V_{1}(t) & \leq 2 t\left[\int_{0}^{t_{0}}+\int_{t_{0}}^{t}\right]\left[\int_{0}^{\infty} \bar{F}_{Z}(v)\left[\sum_{k=1}^{\infty} c(k) \mathbb{P}\left(Z>y+v+T_{k}\right)\right] d v\right] d y \\
& \leq 2 t t_{0}\left(\mathbb{E}(Z) \mathbb{E}(G(Z))+C_{1} t^{2 H-2+\epsilon}\right) \leq C_{2} t, \quad t \geq 1 .
\end{aligned}
$$

Note, $\min (x, y)^{2} \leq x y$ for $x, y \geq 0$. Hence,

$$
\begin{aligned}
V_{2}(t) & =\int_{\mathbb{R}_{+}} \int_{\mathbb{R}} \mathbf{1}_{\{s \leq 0\}}\left((s+z)_{+} \wedge t\right)^{2} d s F_{Z}(d z) \leq \int_{\mathbb{R}_{+}} \int_{-\infty}^{0}(s+z)_{+} t d s F_{Z}(d z) \\
& =t \int_{\mathbb{R}_{+}} \int_{-z}^{0}(s+z) d s F_{Z}(d z)=t \int_{\mathbb{R}_{+}} \frac{z^{2}}{2} F_{Z}(d z)=t \frac{\mathbb{E}\left(Z^{2}\right)}{2} .
\end{aligned}
$$

(b) With the notation of (6.6) and $f(s, z)=g(s, z)=\mathbf{1}_{\{0<s \leq t\}}(s+z-t)_{+}$we have

$$
\operatorname{Var}\left(A^{(2)}(t)\right)=V_{1}(t)+V_{2}(t) .
$$

First,

$$
\begin{aligned}
& V_{1}(t) \\
& \left.=\sum_{\substack{k=-\infty \\
k \neq 0}}^{\infty} c(|k|) \int_{\mathbb{R} \times \mathbb{R}_{+}^{2}} \mathbb{E}\left(\mathbf{1}_{\left\{0<s \leq t, 0<s+T_{k} \leq t\right\}}\left(s+z_{1}-t\right)_{+}\left(s+T_{k}+z_{2}-t\right)\right)_{+}\right) F_{Z}\left(d z_{1}\right) F_{Z}\left(d z_{2}\right) d s \\
& =2 \sum_{k=1}^{\infty} c(k) \int_{[0, t] \times \mathbb{R}_{+}^{2}} \int_{\mathbb{R}_{+}^{2}} \mathbb{E}\left(\mathbf{1}_{\left\{s+T_{k} \leq t\right\}} \mathbf{1}_{\left\{x<s+z_{1}-t\right\}} \mathbf{1}_{\left\{y<s+T_{k}+z_{2}-t\right\}}\right) d x d y F_{Z}\left(d z_{1}\right) F_{Z}\left(d z_{2}\right) d s \\
& =2 \int_{0}^{t}\left[\int_{\mathbb{R}_{+}} \bar{F}_{Z}(x+v) d x\right]\left[\int_{\mathbb{R}_{+}} \sum_{k=1}^{\infty} c(k) \mathbb{P}\left(y+v-Z<T_{k} \leq v\right) d y\right] d v .
\end{aligned}
$$

Markov's inequality gives

$$
\int_{0}^{\infty} \bar{F}_{Z}(x+v) d x \leq \mathbb{E}\left(Z^{2}\right) \int_{0}^{\infty} \frac{1}{(x+v)^{2}} d x \leq C_{1} v^{-1}
$$

Further,

$$
\begin{aligned}
\sum_{k=1}^{\infty} c(k) & \mathbb{P}\left(y+v-Z<T_{k} \leq v\right) \\
= & \int_{y}^{y+v} \sum_{k=1}^{\infty} c(k) \mathbb{P}\left(y+v-z<T_{k} \leq v\right) F_{Z}(d z)+\int_{y+v}^{\infty} \sum_{k=1}^{\infty} c(k) \mathbb{P}\left(T_{k} \leq v\right) F_{Z}(d z) \\
= & {\left[\int_{y}^{y+v / 2}+\int_{y+v / 2}^{y+v}\right][G(v)-G(y+v-z)] F_{Z}(d z)+\int_{y+v}^{\infty} G(v) F_{Z}(d z) } \\
=: & J_{1}(y, v)+J_{2}(y, v)+J_{3}(y, v) .
\end{aligned}
$$

Since $G \in \mathcal{R}_{2 H-1}$ we have for large $v$ by Lemma 2.3 and Lemma 6.1 (a) that

$$
\begin{aligned}
J_{1}(y, v) & \leq C_{2} G(v) v^{-1} \int_{y}^{y+v / 2}(z-y) F_{Z}(d z) \leq C_{2} G(v) v^{-1} \frac{v}{2} \mathbb{P}(Z>y) \\
& \leq C_{3} G(v) y^{-2} \mathbf{1}_{\{y \geq 1\}}+C_{3} G(v) \mathbf{1}_{\{0 \leq y \leq 1\}} .
\end{aligned}
$$


Note that by the monotonicity of $G$ and Markov's inequality we obtain

$$
J_{2}(y, v) \leq G(v) \mathbb{P}(Z>y+v / 2) \leq C_{4} G(v)(y+v / 2)^{-2} .
$$

Finally,

$$
J_{3}(y, v)=G(v) \mathbb{P}(Z>y+v) \leq C_{5} G(v)(y+v)^{-2} .
$$

Thus, (6.44)-(6.46) result in

$$
\int_{0}^{\infty}\left[J_{1}(y, v)+J_{2}(y, v)+J_{3}(y, v)\right] d y \leq C_{6} G(v)\left[\int_{1}^{\infty} \frac{1}{y^{2}} d y+1 \int_{0}^{1} \mathbf{1} d y\right] \leq C_{7} G(v)
$$

for $v \geq v_{0}$ and some $v_{0}>1$. Since $G \in \mathcal{R}_{2 H-1}$ we obtain by Karamata's Theorem and (6.41), (6.42), (6.43), (6.47),

$$
\begin{aligned}
V_{1}(t) & =2\left[\int_{0}^{v_{0}}+\int_{v_{0}}^{t}\right]\left[\int_{\mathbb{R}_{+}} \bar{F}_{Z}(x+v) d x\right]\left[\int_{\mathbb{R}_{+}} \sum_{k=1}^{\infty} c(k) \mathbb{P}\left(y+v-Z<T_{k} \leq v\right) d y\right] d v \\
& \leq C_{8}+C_{9} \int_{v_{0}}^{t} G(v) v^{-1} d v \sim C_{10} G(t) \quad \text { as } t \rightarrow \infty
\end{aligned}
$$

Finally,

$$
V_{2}(t)=\lambda \int_{\mathbb{R}_{+}} \int_{\mathbb{R}} \mathbf{1}_{\{0<s \leq t\}}(s+z-t)_{+}^{2} d s F_{Z}(d z) \leq \lambda \int_{\mathbb{R}_{+}} \int_{0}^{t} z^{2} d s F_{Z}(d z)=t \lambda \mathbb{E}\left(Z^{2}\right) .
$$

Hence, (6.48) and (6.49) complete the proof.

\section{Proof of Theorem 5.5.}

(a) follows again by Lemma 6.2 (b), and Mikosch and Samorodnitsky [26], Theorem 4.2.

(b) We use again the decomposition given in (6.32). On the one hand, applying Theorem 4.1 we have that

$$
\left(\frac{\sum_{i=1}^{n}\left[N_{i}\left(0, M_{n} t\right]-\lambda M_{n} t\right]}{\sqrt{n \operatorname{Var}\left(N\left(0, M_{n}\right]\right)}}\right)_{t \geq 0} \Longrightarrow\left(B_{H}(t)\right)_{t \geq 0} \quad \text { as } n \rightarrow \infty \text { in } \mathbb{C}[0,1] .
$$

Furthermore, note that $\gamma<3-2 H$ is equivalent to

$$
\frac{2-\gamma}{2 H \gamma-2}>\frac{1}{\gamma-1}
$$

We use now the fact that if $\gamma>H^{-1}$ then $\gamma^{-1}(2 H \gamma-2)>0$. Moreover, $\operatorname{Var}(N(0, \cdot]) \in \mathcal{R}_{2 H}$ by Proposition 3.1. Thus, by Potter's Theorem for any $\epsilon>0$ there exist $C_{1}>0$ and $n_{0} \in \mathbb{N}$ such that for $n \geq n_{0}$ we have

$$
\begin{aligned}
\frac{n \operatorname{Var}\left(N\left(0, M_{n}\right]\right)}{a\left(n M_{n}\right)^{2}} & \leq C_{1} \frac{n M_{n}^{2 H+\epsilon}}{\left(n M_{n}\right)^{\frac{2}{\gamma}-\epsilon}} \\
& =C_{1} n^{-\frac{2-\gamma}{\gamma}} M_{n}^{\frac{2 H \gamma-2}{\gamma}} n^{\epsilon} M_{n}^{2 \epsilon} \\
& =C_{1}\left(n^{\frac{2-\gamma}{2 H \gamma-2}} M_{n}^{-1}\right)^{-\frac{2 H \gamma-2}{\gamma}-2 \epsilon} n^{\epsilon+2 \epsilon \frac{2-\gamma}{2 H \gamma-2}}
\end{aligned}
$$


which tends to 0 as $n \rightarrow \infty$ for $\epsilon$ small enough by (5.2). Hence, Corollary 13.3.2 of Whitt [34], (6.50), (6.35) and (6.51) result in

$$
\left(\frac{\sum_{l=1}^{\sum_{i=1}^{n} N_{i}\left(0, M_{n} t\right]} \mathcal{Z}_{l}-\lambda n M_{n} t \mathbb{E}(Z)}{a\left(n M_{n}\right)}\right)_{t \geq 0} \Longrightarrow\left(\lambda^{\frac{1}{\gamma}} S_{\gamma}(t)\right)_{t \geq 0} \text { as } n \rightarrow \infty \text { in }\left(\mathbb{D}[0,1], J_{1}\right) \text {. }
$$

A conclusion of (6.34) and (6.52) is that

$$
\left(\frac{S_{n, M_{n}}^{(1)}(t)}{a\left(n M_{n}\right)}\right)_{t \geq 0} \Longrightarrow\left(\lambda^{\frac{1}{\gamma}} S_{\gamma}(t)\right)_{t \geq 0} \text { as } n \rightarrow \infty \text { in }\left(\mathbb{D}[0,1], J_{1}\right)
$$

Return to mind that $\mathbb{E}\left(A^{(j)}(\cdot)\right) \in \mathcal{R}_{2-\gamma}$ by Lemma $6.4, j=1,2$. Thus, by Potter's Theorem for any $\epsilon>0$ there exist $C_{2}>0$ and $n_{0} \in \mathbb{N}$ such that for $n \geq n_{0}$ we have

$$
\begin{aligned}
\frac{n \mathbb{E}\left(A^{(j)}\left(M_{n} t\right)\right)}{a\left(n M_{n}\right)} & \leq C_{2} \frac{n\left(M_{n} t\right)^{2-\gamma+\epsilon}}{\left(n M_{n}\right)^{\frac{1}{\gamma}-\epsilon}}=C_{2} n^{\frac{\gamma-1}{\gamma}} M_{n}^{-\frac{1}{\gamma}(\gamma-1)^{2}} n^{\epsilon} M_{n}^{2 \epsilon} t^{2-\gamma+\epsilon} \\
& =C_{2} n^{\epsilon+\frac{2 \epsilon}{\gamma-1}}\left(n^{\frac{1}{\gamma-1}} M_{n}^{-1}\right)^{\frac{(\gamma-1)^{2}}{\gamma}-2 \epsilon} t^{2-\gamma+\epsilon},
\end{aligned}
$$

which tends to 0 as $n \rightarrow \infty$ for $\epsilon$ small enough by (5.1). Thus, Markov's inequality gives

$$
\mathbb{P}\left(\frac{1}{a\left(n M_{n}\right)} \sum_{i=1}^{n} A_{i}^{(j)}\left(M_{n} t\right)>\delta\right) \leq \frac{n \mathbb{E}\left(A^{(j)}\left(M_{n} t\right)\right)}{\epsilon a\left(n M_{n}\right)} \longrightarrow 0 \quad \text { as } n \rightarrow \infty \text { for any } \delta>0,
$$

and finally for $t>0$,

$$
\frac{S_{n, M_{n}}^{(2)}(t)}{a\left(n M_{n}\right)} \stackrel{\mathbb{P}}{\longrightarrow} 0 \quad \text { as } n \rightarrow \infty .
$$

The statement in (b) is then a conclusion of (6.32), (6.53) and (6.55).

(c) The conclusion follows from (6.32), (6.35), (6.50), (6.54), Whitt [34], Corollary 13.3.2, and

$$
\frac{a\left(n M_{n}\right)^{2}}{n \operatorname{Var}\left(N\left(0, M_{n}\right]\right)} \leq C_{3}\left(n^{\frac{2-\gamma}{2 H \gamma-2}} M_{n}^{-1}\right)^{\frac{2 H \gamma-2}{\gamma}-(2 \epsilon)} n^{\epsilon+2 \epsilon \frac{2-\gamma}{2 H \gamma-2}}
$$

which tends to 0 as $n \rightarrow \infty$ for $\epsilon$ small enough by the small growth condition.

\section{Proof of Theorem 5.6.}

(a) We use the arguments as in Theorem 5.6 (a).

(b) On the one hand, $\gamma<2$ implies $1-2 \gamma^{-1}<0$. On the other hand, since $\gamma<H^{-1}$ we have $2\left(H-\gamma^{-1}\right)<0$. Hence, again by Potter's Theorem for any $\epsilon>0$ there exist $C_{1}>0$ and $n_{0} \in \mathbb{N}$ such that for $n \geq n_{0}$

$$
\frac{n \operatorname{Var}\left(N\left(0, M_{n}\right]\right)}{a\left(n M_{n}\right)^{2}} \leq C_{1} \frac{n M_{n}^{2 H+\epsilon}}{\left(n M_{n}\right)^{\frac{2}{\gamma}-\epsilon}}=C_{1} n^{1-\frac{2}{\gamma}+\epsilon} M_{n}^{2\left(H-\gamma^{-1}\right)+2 \epsilon}
$$

which tends to 0 as $n \rightarrow \infty$ for $\epsilon$ small enough. Note, that here we not require the slow growth condition. Then the conclusion follows from (6.32), (6.35), (6.50), (6.54), Whitt [34], Corollary 13.3.2 and (6.56). 


\section{References}

[1] Anderson, K. K. And Athreya, K. B. (1988). A strong renewal theorem for generalized renewal functions in the infinite mean case. Probab. Th. Rel. Fields 77, 471-479.

[2] Baccelli, F. And Brémaud, P. (2003). Elements of Queueing Theory: Palm Martingale Calculus and Stochastic Recurrences. Springer, Heidelberg.

[3] Billingsley, P. (1968). Convergence of Probability and Measures. 1st edn. Wiley, New York.

[4] Billingsley, P. (1986). Probability and Measure. 2nd edn. Wiley, New York.

[5] Billingsley, P. (1999). Convergence of Probability and Measures. 2nd edn. Wiley, New York.

[6] Bingham, N. H., Goldie, C. M., And Teugels, J. L. (1987). Regular Variation. Cambridge University Press, Cambridge.

[7] Crovella, M. and Bestavros, A. (1995). Explaining world wide web traffic selfsimilarity. Preprint.

[8] Crovella, M. and Bestavros, A. (1997). Self-similarity in world wide web traffic: evidence and possible causes. IEEE/ACM Trans. Networking 5, 835-846.

[9] Crovella, M., Taqqu, M., and Bestavros, A. (1999). Heavy-tailed probability distributions in the world wide web. In: R. Adler and R. Epstein (Eds.), A Practical Guide to Heavy Tails: Statistical Techniques for Analysing Heavy Tailed Distributions, pp. 3-26. Birkhäuser, Boston.

[10] Daley, D. J. and Vere-Jones, D. (2003). An Introduction to the Theory of Point Processes. Vol I: Elementary Theory and Methods. 2nd edn. Springer, New York.

[11] Daley, D. J. and Vere-Jones, D. (2008). An Introduction to the Theory of Point Processes. Vol II: General Theory and Structure. 2nd edn. Springer, New York.

[12] Doney, R. (1997). One-sided local large deviation and renewal theorems in the case of infinite mean. Probab. Theory Related Fields 107, 451-465.

[13] Embrechts, P., Klüppelberg, C., And Mikosch, T. (1997). Modelling Extremal Events for Insurance and Finance. Springer, Berlin.

[14] Fä̈, G., González-Arévalo, B., Mikosch, T., and Samorodnitsky, G. (2006). Modeling teletraffic arrivals by a Poisson cluster process. Queueing System 54, 121-140.

[15] Fasen, V. and Samorodnitsky, G. (2009). A fluid cluster Poisson input process can look like a fractional Brownian motion even in the slow growth aggregation regime. Adv. Appl. Probab. 41, 393-427. 
[16] Gaigalas, R. And KaJ, I. (2003). Convergence of scaled renewal processes and a packet arrival model. Bernoulli 9, 671-703.

[17] Guerin, C., Nyberg, H., Perrin, O., Resnick, S., Rootzén, H., and Stărică, C. (2003). Empirical testing of the infinite source Poisson data traffic model. Stochastic Models 19, 151-200.

[18] Hernandes-Campos, H., Marron, S., Samorodnitsky, G., and Smith, F. (2004). Variable heavy tailed durations in Internet traffic. Performance Eval. 58, 261-284.

[19] KaJ, I. And TAqQU, M. S. (2008). Convergence to fractional Brownian motion and to the Telecom process: the integral representation approach. In: M. E. Vares and V. Sidoravicius (Eds.), Out of Equilibrium 2, vol. 60 of Progress in Probability, pp. 383-427. Birkhäuser.

[20] Kallenberg, O. (2002). Foundations of Modern Probability. 2nd edn. Springer, New York.

[21] KarR, A. F. (1986). Point processes and their statistical inference. Marcel Decker, New York.

[22] Leland, W. E., Taqqu, M. S., Willinger, W., and Wilson, D. V. (1994). On the self-similar nature of Ethernet traffic (extended version). IEEE/ACM Trans. Networking 2, $1-15$.

[23] Levy, J. AND TAqQU, M. S. (1987). On renewal processes having stable inter-renewal intervals and stable rewards. Ann. Sci. Math. Québec 11, 95-110.

[24] Levy, J. And Taqqu, M. S. (2000). Renewal reward processes with heavy-tailed interrenewal intervals and stable rewards. Bernoulli 6, 23-44.

[25] Mikosch, T., Resnick, S., Rootzén, H., and Stegeman, A. (2002). Is network traffic approximated by stable Lévy motion or fractional Brownian motion? Ann. Appl. Probab. 12, 23-68.

[26] Mikosch, T. And Samorodnitsky, G. (2007). Scaling limits for cumulative input processes. Math. Oper. Res. 32, 890-919.

[27] Omey, E. (1982). Multivariate Regular Variation and its Applications in Probability Theory. Ph.D. thesis, University of Leuven.

[28] Pipiras, V. And Taqqu, M. (2004). Slow, fast and arbitrary growth conditions for renewalreward processes when both the renewals and the rewards are heavy-tailed. Bernoulli 10, $121-163$.

[29] Resnick, S. and Van den Berg, E. (2000). Convergence of high-speed network traffic models. J. Appl. Probab. 37, 575-597. 
[30] Resnick, S. I. (2007). Heavy-Tail Phenomena: Probabilistic and Statistical Modeling. Springer, New York.

[31] Sato, K. (1999). Lévy Processes and Infinitely Divisible Distributions. Cambridge University Press, Cambridge.

[32] Taqqu, M. S. And Levy, J. (1986). Using renewal processes to generate long-range dependence and high variability. In: E. Eberlein and M. S. Taqqu (Eds.), Dependence in Probability and Statistics, pp. 73-89. Birkhäuser, Boston.

[33] Whitт, W. (1999). Limits for cumulative input processes to queues. Tech. rep., AT\&T Labs.

[34] Whitt, W. (2002). Stochastic-Process Limits: An Introduction to Stochastic-Process Limits and Their Applications to Queues. Springer, New York.

[35] Willinger, W., Taqqu, M., Sherman, R., and Wilson, D. (1997). Self-similarity through high-variability: statistical analysis of Ethernet LAN traffic at the source level. IEEE/ACM Trans. Networking 5, 71-86.

[36] Zolotarev, V. (1957). Mellin-Stieltjes transforms in probability theory. Theory Probab. Appl. 2, 433-459. 